\title{
Types of Submanifolds in Metallic Riemannian Manifolds: A Short Survey
}

\author{
Cristina E. Hretcanu ${ }^{1, *,+(\mathbb{D})}$ and Adara M. Blaga ${ }^{2,+}$ (D) \\ 1 Faculty of Food Engineering, Stefan cel Mare University of Suceava, 720229 Suceava, Romania \\ 2 Faculty of Mathematics and Computer Science, West University of Timisoara, 300223 Timisoara, Romania; \\ adara.blaga@e-uvt.ro \\ * Correspondence: cristina.hretcanu@fia.usv.ro \\ + These authors contributed equally to this work.
}

Citation: Hretcanu, C.E.; Blaga, A.M Types of Submanifolds in Metallic Riemannian Manifolds: A Short Survey. Mathematics 2021, 9, 2467. https://doi.org/10.3390/math9192467

Academic Editor: Juan Benigno Seoane-Sepúlveda

Received: 2 September 2021

Accepted: 23 September 2021

Published: 3 October 2021

Publisher's Note: MDPI stays neutral with regard to jurisdictional claims in published maps and institutional affiliations.

Copyright: (c) 2021 by the authors. Licensee MDPI, Basel, Switzerland. This article is an open access article distributed under the terms and conditions of the Creative Commons Attribution (CC BY) license (https:// creativecommons.org/licenses/by/ $4.0 /)$.

\begin{abstract}
We provide a brief survey on the properties of submanifolds in metallic Riemannian manifolds. We focus on slant, semi-slant and hemi-slant submanifolds in metallic Riemannian manifolds and, in particular, on invariant, anti-invariant and semi-invariant submanifolds. We also describe the warped product bi-slant and, in particular, warped product semi-slant and warped product hemi-slant submanifolds in locally metallic Riemannian manifolds, obtaining some results regarding the existence and nonexistence of non-trivial semi-invariant, semi-slant and hemi-slant warped product submanifolds. We illustrate all these by suitable examples.
\end{abstract}

Keywords: metallic Riemannian manifold; warped product submanifold; slant; semi-slant; hemi-slant; bi-slant submanifold

MSC: 53B20; 53B25; 53C42; 53C15

\section{Introduction}

The notion of Golden structure on a Riemannian manifold was introduced by C. E. Hretcanu and M. Crasmareanu in ([1]). Then, the properties of submanifolds in Golden Riemannian manifolds were studied in $([2,3])$ using the corespondents of a Golden structure related to an almost product structure. The metallic structure introduced in ([4]) is a generalization of the Golden structure. Different types of submanifolds in metallic and Golden Riemannian manifolds were studied in ([5-7]), obtaining different integrability conditions for the distributions involved in these types of submanifolds. The metallic (and in particular Golden) warped product Riemannian manifold was studied in ([8-10]).

In this paper we provide a brief survey on the properties of metallic structures defined on Riemannian manifolds, stating the definitions and some properties of these structures, related to generalized secondary Fibonacci sequences (in Section 2). Then, in Section 3, we discuss about some properties of the structures induced on submanifolds, called by us, $\Sigma$-metallic Riemannian structures, especially regarding the normality of these types of structures and, in particular, we present some properties of invariant and anti-invariant submanifolds in metallic Riemannian manifolds ([11]). In Section 4, we define and discuss about properties of slant submanifolds in metallic Riemannian manifolds. In Section 5, we treat bi-slant submanifolds in metallic Riemannian manifolds and, in particular, for semi-slant and hemi-slant submanifolds, we obtain some integrability conditions of the distributions involved. In Section 6, we give some properties of warped product submanifolds in metallic Riemannian manifolds and provide examples of warped product semi-invariant, semi-slant and respectively hemi-slant submanifolds in these types of manifolds. 


\section{Preliminaries}

We recall the definitions and some basic formulas for metallic structures on a Riemannian manifold and, in particular, for Golden Riemannian structures.

The name of "metallic means family" (MMF) (or metallic proportions) was introduced by Vera W. de Spinadel ([12]) as the set of positive solutions $\sigma_{p, q}=\frac{p+\sqrt{p^{2}+4 q}}{2}$ of the equation $x^{2}-p x-q=0$, where $p$ and $q$ are positive integer values. These $\sigma_{p, q}$ numbers can be seen as generalizations of the Golden number $\phi=\frac{1+\sqrt{5}}{2}=1.618 \ldots$ (the positive solution of the equation $x^{2}-x-1=0$ ). The name metallic means family (MMF) was explained by Vera W. de Spinadel in ([13]) where she affirmed that "besides carrying the name of a metals, they have common mathematical properties that attach a fundamental importance to them in modern investigations about the search of universal roads to chaos." These metallic numbers found many applications in researches that "analyze the behavior of non linear dynamical systems when they proceed from a periodic regime to a chaotic one" ([14]). Moreover, Vera W. de Spinadel ([15]) remarked that the metallic means family is the set of positive eigenvalues of the matrix $\left(\begin{array}{ll}p & q \\ 1 & 0\end{array}\right)$ for different values of natural number $p$ and integer $q$, with $p^{2}+4 q>0$.

The generalized secondary Fibonacci sequence $([12,16,17])$ are given by relations of the type

$$
G_{n+1}=p \cdot G_{n}+q \cdot G_{n-1}, \quad n \geq 1,
$$

where $p, q, G_{0}=a$ and $G_{1}=b$ are real numbers. The ratio $G_{n+1} / G_{n}$ of two consecutive generalized secondary Fibonacci numbers converges to the metallic numbers $\sigma_{p, q}$ which in particular are called: the Golden mean $\phi=\frac{1+\sqrt{5}}{2}$ (for $a=b=p=q=1$ ), the silver mean $\sigma_{2,1}=1+\sqrt{2}$, the bronze mean $\sigma_{3,1}=\frac{3+\sqrt{13}}{2}$, the subtle mean $\sigma_{4,1}=2+\sqrt{5}=\phi^{3}$, the copper mean $\sigma_{1,2}=2$, the nickel mean $\sigma_{1,3}=\frac{1+\sqrt{13}}{2}$ and so on.

The most remarkable element from (MMF) is the Golden mean, known from ancient times as an expression of harmony of many constructions, paintings and in music. It also appears as an expression of the objects from the natural world (flowers, trees, fruits) that possess pentagonal symmetry ([18]).

One can remark that Golden mean is determined by the ratio of two consecutive classical Fibonacci numbers and the silver mean is determined by the ratio of two consecutive Pell numbers ([19]). The bronze mean plays an important role in studying topics such as dynamical systems and quasicrystals and the subtle mean is significant in the theory of Cantorian fractal-like micro-space-time $E^{\infty}$, being involved, in a fundamental way, in noncommutative geometry and four manifold theory $([20,21])$.

The members of (MMF), named also metallic numbers, have found many applications in differential geometry, as it can be seen in the next properties.

A metallic structure on a differentiable manifold is a particular case of a polynomial structure introduced by S. I. Goldberg, K. Yano and N. C. Petridis in ([22,23]).

Precisely, a polynomial structure $J$ of degree 2, defined on a differentiable manifold $\bar{M}$, is called a metallic structure if it satisfies the equality $J^{2}=p \cdot J+q \cdot I$, where $I$ is the identity endomorphism on $T \bar{M}$ and $p, q \in N^{*}$. The pair $(\bar{M}, J)$ is called a metallic manifold.

Another important (for our study) is the polynomial structure $F$ of degree 2 on $\bar{M}$, i.e., $F^{2}=I$, called an almost product structure. In this case, $(\bar{M}, F)$ is called an almost product manifold.

The metallic structure and the almost product structure are closely related shown below.

Proposition 1 ([4]). (i) Any metallic structure J induces two almost product structures, given by

$$
F_{1,2}= \pm \frac{2}{2 \sigma_{p, q}-p} \cdot J-\frac{p}{2 \sigma_{p, q}-p} \cdot I .
$$


(ii) Any almost product structure F induces two metallic structures, given by

$$
J_{1,2}= \pm \frac{2 \sigma_{p, q}-p}{2} \cdot F+\frac{p}{2} \cdot I
$$

Definition 1. A triple $(\bar{M}, \bar{g}, J)$ is called a metallic Riemannian manifold if the differentiable manifold $\bar{M}$ is endowed with a metallic structure J and a Riemannian metric $\bar{g}$ such that

$$
\bar{g}(J X, Y)=\bar{g}(X, J Y)
$$

for any $X, Y \in \Gamma(T \bar{M})$.

We remark that in a metallic Riemannian manifold $(\bar{M}, \bar{g}, J)$, we have

$$
\bar{g}(J X, J Y)=p \bar{g}(J X, Y)+q \bar{g}(X, Y)
$$

for any $X, Y \in \Gamma(T \bar{M})$.

Recall that an almost product Riemannian structure $(\bar{g}, F)$ on $\bar{M}$ is a pair $(\bar{g}, F)$, where $\bar{g}$ is a Riemannian metric and $F$ is a $\bar{g}$-compatible almost product structure on $\bar{M}$, i.e.,

$$
\bar{g}(F X, Y)=\bar{g}(X, F Y)
$$

for any $X, Y \in \Gamma(T \bar{M})$. In this case, $(\bar{M}, \bar{g}, F)$ is called almost product Riemannian manifold.

If the almost product structure $F$ is a Riemannian one, then the induced metallic structures $J_{1}$ and $J_{2}$ are metallic Riemannian structures.

On a metallic manifold $(\bar{M}, J)$ there are two complementary distributions $\mathcal{D}_{1}$ and $\mathcal{D}_{2}$ corresponding to the projection operators $\pi_{1}$ and $\pi_{2}([4])$

$$
\pi_{1}=-\frac{1}{2 \sigma_{p, q}-p} \cdot J+\frac{\sigma_{p, q}}{2 \sigma_{p, q}-p} \cdot I, \quad \pi_{2}=\frac{1}{2 \sigma_{p, q}-p} \cdot J+\frac{\sigma_{p, q}-p}{2 \sigma_{p, q}-p} \cdot I,
$$

which verify the following relations

$$
\begin{gathered}
\pi_{1}+\pi_{2}=I, \quad \pi_{1}^{2}=\pi_{1}, \quad \pi_{2}^{2}=\pi_{2}, \quad \pi_{1} \pi_{2}=\pi_{2} \pi_{1}=0, \\
J \pi_{1}=\pi_{1} J=\left(p-\sigma_{p, q}\right) \cdot \pi_{1}, \quad J \pi_{2}=\pi_{2} J=\sigma_{p, q} \cdot \pi_{2} .
\end{gathered}
$$

Thus

$$
J=\left(p-\sigma_{p, q}\right) \cdot \pi_{1}+\sigma_{p, q} \cdot \pi_{2}
$$

Proposition 2 ([4]). A metallic structure J on $\bar{M}$ has the following properties:

(i) for every integer number $n \geq 1$ :

$$
J^{n}=G_{n} \cdot J+q G_{n-1} \cdot I
$$

where $\left(G_{n}\right)_{n \in N^{*}}$ is the generalized secondary Fibonacci sequence with $G_{0}=0$ and $G_{1}=1$;

(ii) $J$ is an isomorphism on $T_{x} \bar{M}$, for every $x \in \bar{M}$. It follows that $J$ is invertible and its inverse $\hat{J}=J^{-1}=\frac{1}{q} \cdot J-\frac{p}{q} \cdot I$ is not a metallic structure, but it is still polynomial, more precisely, $a$ quadratic one:

$$
q \cdot \hat{J}^{2}+p \cdot \hat{J}-I=0
$$

(iii) the eigenvalues of $J$ are the metallic number $\sigma_{p, q}$ and $p-\sigma_{p, q}$.

In particular, for $p=q=1$ one gets the Golden structure ([24,25]), namely a $(1,1)$ tensor field $J$ which satisfies $J^{2}=J+I$. In this case, $(\bar{M}, J)$ is called a Golden manifold. Every Golden structure defines two almost product structures and any almost product structure defines two Golden structures $([2,24])$. The eigenvalues of $J$ are the Golden Ratio 
$\phi$ and $1-\phi$. Notice that the Golden structures appear in pairs, i.e., if $J$ is a Golden structure, then $\tilde{J}=I-J$ is also a Golden structure.

Moreover, the power $n \geq 1$ of the Golden structure $J$ on $\bar{M}$ can be written using the Fibonacci sequence $\left(f_{n}\right)_{n \in N^{*}}([24])$

$$
J^{n}=f_{n} \cdot J+f_{n-1} \cdot I .
$$

By using an explicit expression for the Fibonacci sequence, namely, the Binet's formula from ([18])

$$
f_{n}=\frac{\phi^{n}-(1-\phi)^{n}}{\sqrt{5}}
$$

we can obtain an expression of the power $n \geq 1$ of the Golden structure $J$ by means of the Golden number $\phi$ ([24]).

\section{Submanifolds in Metallic Riemannian Manifolds}

In ([11]), C. E. Hretcanu and A. M. Blaga discussed about some properties of the structure induced by a metallic Riemannian structure on a submanifold, called $\Sigma$-metallic Riemannian structure.

Let $M^{m}$ be an $m$-dimensional Riemannian manifold with the metric $g$ and let $\Gamma(T M)$ be the set of all vector fields on $M$.

Definition 2. If on $M^{m}$ there exist a tensor field $T$ of type $(1,1), r$ vector fields $\xi_{1}, \ldots, \xi_{r}(0<r<m)$, $r$-forms $u_{1}, \ldots, u_{r}$ and a $r \times r$ matrix $\left(a_{\alpha \beta}\right)_{r}(\alpha, \beta \in\{1, \ldots, r\})$ of differentiable functions which verify the relations

$$
\begin{gathered}
u_{\alpha}(X)=g\left(X, \xi_{\alpha}\right), \\
T^{2} X=p T X+q X-\sum_{\alpha=1}^{r} u_{\alpha}(X) \xi_{\alpha}, \\
u_{\alpha}(T X)=p u_{\alpha}(X)-\sum_{\beta=1}^{r} a_{\alpha \beta} u_{\beta}(X), \quad a_{\alpha \beta}=a_{\beta \alpha}, \\
u_{\beta}\left(\xi_{\alpha}\right)=q \delta_{\alpha \beta}+p a_{\alpha \beta}-\sum_{\gamma=1}^{r} a_{\alpha \gamma} a_{\gamma \beta}, \quad T \xi_{\alpha}=p \xi_{\alpha}-\sum_{\beta=1}^{r} a_{\alpha \beta} \xi_{\beta},
\end{gathered}
$$

for any $X \in \Gamma(T M)$, where $\delta_{\alpha \beta}$ is the Kronecker symbol and $p, q$ are positive integers, then the structure $\Sigma=\left(T, g, u_{\alpha}, \xi_{\alpha},\left(a_{\alpha \beta}\right)_{r}\right)$ is called a $\Sigma$-metallic Riemannian structure ([11]).

In particular, for $p=q=1$, the structure $\Sigma=\left(T, g, u_{\alpha}, \xi_{\alpha},\left(a_{\alpha \beta}\right)_{r}\right)$ is called a $\Sigma$-Golden Riemannian structure.

If $M$ is a submanifold of dimension $m \in N^{*}$, isometrically immersed in a metallic Riemannian manifold $(\bar{M}, \bar{g}, J), T_{x} M$ is the tangent space of $M$ in a point $x \in M$ and $T_{x}^{\perp} M$ is the normal space of $M$ in $x$, then $T_{x} \bar{M}=T_{x} M \oplus T_{x}^{\perp} M$, for any $x \in M$.

Denoting by $i_{*}$ the differential of the immersion $i: M \rightarrow \bar{M}$ and by $X$ the vector field $i_{*} X$, for $X \in \Gamma(T M)$, the induced Riemannian metric $g$ on $M$ is given by $g(X, Y)=$ $\bar{g}\left(i_{*} X, i_{*} Y\right)$, for any $X, Y \in \Gamma(T M)$.

Let us consider, for any $X \in \Gamma(T M)$ and $V \in \Gamma\left(T^{\perp} M\right)$, the decomposition of $J X$ and $J V$ into the tangential and normal components

$$
J X=T X+N X, \quad J V=t V+n V,
$$

where $T X:=(J X)^{\top}, N X:=(J X)^{\perp}, t V:=(J V)^{\top}$ and $n V:=(J V)^{\perp}$. 
It can be easily checked that the maps $T$ and $n$ are $\bar{g}$-symmetric ([5])

$$
\bar{g}(T X, Y)=\bar{g}(X, T Y), \quad \bar{g}(n U, V)=\bar{g}(U, n V),
$$

for any $X, Y \in \Gamma(T M)$ and $U, V \in \Gamma\left(T^{\perp} M\right)$. Moreover:

$$
\bar{g}(N X, U)=\bar{g}(X, t U),
$$

for any $X \in \Gamma(T M)$ and $U \in \Gamma\left(T^{\perp} M\right)$.

If $r \in N^{*}$ is the codimension of the submanifold $M$ in the Riemannian manifold $(\bar{M}, \bar{g})$, then we can fix a local orthonormal basis $\left\{N_{1}, \ldots, N_{r}\right\}$ of $T^{\perp} M$. Hereafter we assume that the indices $\alpha, \beta, \gamma$ run over the range $\{1, \ldots, r\}$.

Then the vectors $J X$ and $J N_{\alpha}$ can be decomposed into the tangential and normal components ([4])

$$
J X=T X+\sum_{\alpha=1}^{r} u_{\alpha}(X) N_{\alpha}, \quad J N_{\alpha}=\xi_{\alpha}+\sum_{\beta=1}^{r} a_{\alpha \beta} N_{\beta},
$$

for any $X \in \Gamma(T M)$, where $\xi_{\alpha}=: t N_{\alpha}$ are vector fields on $M, u_{\alpha}$ are 1-forms on $M,\left(a_{\alpha \beta}\right)_{r}$ is an $r \times r$ matrix of differentiable functions on $M, \alpha, \beta \in\{1, \ldots, r\}$, and

$$
N X=\sum_{\alpha=1}^{r} u_{\alpha}(X) N_{\alpha}, \quad n N_{\alpha}=\sum_{\beta=1}^{r} a_{\alpha \beta} N_{\beta}
$$

We can remark that

Theorem 1 ([11]). The structure $\left(T, g, u_{\alpha}, \xi_{\alpha},\left(a_{\alpha \beta}\right)_{r}\right)$ induced on a submanifold $M$ of codimension $r$ by the metallic Riemannian structure $(\bar{g}, J)$ on $\bar{M}$ satisfies the relations (1)-(4). Thus, it is a $\Sigma$-metallic Riemannian structure.

On the Riemannian manifold $(\bar{M}, \bar{g})$ and its submanifold $(M, g)$, we consider the Levi-Civita connections $\bar{\nabla}$ and $\nabla$, respectively. For any $X, Y \in \Gamma(T M)$ and $V \in \Gamma\left(T^{\perp} M\right)$, the Gauss and Weingarten formulas are given by

$$
\bar{\nabla}_{X} Y=\nabla_{X} Y+h(X, Y), \quad \bar{\nabla}_{X} V=-A_{V} X+\nabla_{X}^{\frac{1}{X}} V,
$$

where $h$ is the second fundamental form, $A_{V}$ is the shape operator of $M$ and $\nabla^{\perp}$ is the normal connection. Moreover:

$$
\bar{g}(h(X, Y), V)=\bar{g}\left(A_{V} X, Y\right),
$$

for any $X, Y \in \Gamma(T M)$ and $V \in \Gamma\left(T^{\perp} M\right)$.

We denote by $A_{N_{\alpha}}=: A_{\alpha}$ and remark that ([11])

$$
\bar{\nabla}_{X} N_{\alpha}=-A_{\alpha} X+\nabla_{X}^{\frac{1}{X}} N_{\alpha}, \quad h_{\alpha}(X, Y)=g\left(A_{\alpha} X, Y\right),
$$

for any $X, Y \in \Gamma(T M), \alpha \in\{1, \ldots, r\}$, where $\left\{N_{1}, \ldots, N_{r}\right\}$ is a local orthonormal basis of the normal space $T_{x}^{\perp} M$ and $r$ is the codimension of $M$ in $\bar{M}$.

For $\alpha \in\{1, \ldots, r\}, \nabla \frac{1}{X} N_{\alpha}=\sum_{\beta=1}^{r} l_{\alpha \beta}(X) N_{\beta}$, for any $X \in \Gamma(T M)$, where $\left(l_{\alpha \beta}\right)_{r}$ is an $r \times r$ matrix of 1 -forms on $M$. Since $\bar{g}\left(N_{\alpha}, N_{\beta}\right)=\delta_{\alpha \beta}$, we obtain ([11])

$$
\bar{g}\left(\nabla_{X}^{\perp} N_{\alpha}, N_{\beta}\right)+\bar{g}\left(N_{\alpha}, \nabla_{X}^{\perp} N_{\beta}\right)=0,
$$


which is equivalent to $l_{\alpha \beta}=-l_{\beta \alpha}$, for any $\alpha, \beta \in\{1, \ldots, r\}$ and $X \in \Gamma(T M)$. From $\bar{g}(J X, Y)=\bar{g}(X, J Y)$, it follows

$$
\bar{g}\left(\left(\bar{\nabla}_{X} J\right) Y, Z\right)=\bar{g}\left(Y,\left(\bar{\nabla}_{X} J\right) Z\right),
$$

for any $X, Y, Z \in \Gamma(T \bar{M})$, where $\bar{\nabla} J$ is the covariant derivative of $J$. Moreover, if $M$ is an isometrically immersed submanifold in the $(\bar{M}, \bar{g}, J)$, then ([9])

$$
\bar{g}\left(\left(\nabla_{X} T\right) Y, Z\right)=\bar{g}\left(Y,\left(\nabla_{X} T\right) Z\right),
$$

for any $X, Y, Z \in \Gamma(T M)$, where $\nabla T$ is the covariant derivative of $T$, given by

$$
\left(\nabla_{X} T\right) Y:=\nabla_{X} T Y-T\left(\nabla_{X} Y\right)
$$

for any $X, Y \in \Gamma(T M)$.

Moreover, the covariant derivatives of $N, t$ and $n$ are, respectively, given by

$$
\begin{gathered}
\left(\bar{\nabla}_{X} N\right) Y:=\nabla_{X}^{\frac{1}{X}} N Y-N\left(\nabla_{X} Y\right), \quad\left(\nabla_{X} t\right) V:=\nabla_{X} t V-t\left(\nabla_{X}^{\frac{1}{X}} V\right), \\
\left(\bar{\nabla}_{X} n\right) V:=\nabla_{X}^{\frac{1}{X}} n V-n\left(\nabla_{X}^{\frac{1}{X}} V\right),
\end{gathered}
$$

for any $X, Y \in \Gamma(T M)$ and $V \in \Gamma\left(T^{\perp} M\right)$.

We can define the notion of locally metallic Riemannian manifold by analogy with a locally product manifold, as follows.

Definition 3. We say that a metallic Riemannian manifold $(\bar{M}, \bar{g}, J)$ is a locally metallic Riemannian manifold if $J$ is parallel with respect to the Levi-Civita connection $\bar{\nabla}$ on $\bar{M}$, i.e., $\bar{\nabla} J=0$.

Proposition 3 ([11]). If $M$ is a submanifold in a locally metallic Riemannian manifold $(\bar{M}, \bar{g}, J)$ with $\nabla$ the Levi-Civita connection on $M$ and $\nabla^{\perp}$ the normal connection, then, for any $X, Y \in$ $\Gamma(T M)$, we have

$$
\begin{gathered}
T([X, Y])=\nabla_{X} T Y-\nabla_{Y} T X-A_{N Y} X+A_{N X} Y, \\
N([X, Y])=h(X, T Y)-h(T X, Y)+\nabla_{X}^{\perp} N Y-\nabla_{Y}^{\perp} N X .
\end{gathered}
$$

Proposition 4 ([11]). If $M$ is a submanifold in a locally metallic Riemannian manifold $(\bar{M}, \bar{g}, J)$, then, for any $X, Y \in \Gamma(T M)$ and $V \in \Gamma\left(T^{\perp} M\right)$, we have

$$
\begin{gathered}
\left(\nabla_{X} T\right) Y=A_{N Y} X+t h(X, Y), \quad\left(\bar{\nabla}_{X} N\right) Y=n h(X, Y)-h(X, T Y), \\
\left(\nabla_{X} t\right) V=A_{n V} X-T A_{V} X, \quad\left(\bar{\nabla}_{X} n\right) V=-h(X, t V)-N A_{V} X .
\end{gathered}
$$

Proposition 5 ([11]). Let $M$ be a submanifold of codimension $r$ in a locally metallic Riemannian manifold $(\bar{M}, \bar{g}, J)$ and let $\left(T, g, u_{\alpha}, \xi_{\alpha},\left(a_{\alpha \beta}\right)_{r}\right)$ be the $\Sigma$-metallic Riemannian structure induced by the metallic Riemannian structure $(\bar{g}, J)$. Then, for any $X, Y \in \Gamma(T M)$, we get

$$
\begin{gathered}
T([X, Y])=\nabla_{X} T Y-\nabla_{Y} T X-\sum_{i=1}^{r}\left[u_{\alpha}(Y) A_{\alpha} X-u_{\alpha}(X) A_{\alpha} Y\right], \\
N([X, Y])=\sum_{\alpha=1}^{n}\left[\left(\nabla_{Y} u_{\alpha}\right) X-\left(\nabla_{X} u_{\alpha}\right) Y+u_{\alpha}(X) l_{\alpha \beta}(Y)-u_{\alpha}(Y) l_{\alpha \beta}(X)\right] N_{\alpha} .
\end{gathered}
$$


Proposition 6 ([11]). Let $M$ be a submanifold of codimension $r$ in a locally metallic Riemannian manifold $(\bar{M}, \bar{g}, J)$ and let $\left(T, g, u_{\alpha}, \xi_{\alpha},\left(a_{\alpha \beta}\right)_{r}\right)$ be the $\Sigma$-metallic Riemannian structure induced by the metallic Riemannian structure $(\bar{g}, J)$. Then, for any $X, Y \in \Gamma(T M)$, we get

$$
\begin{gathered}
\left(\nabla_{X} T\right) Y=\sum_{\alpha=1}^{r} h_{\alpha}(X, Y) \xi_{\alpha}+\sum_{\alpha=1}^{r} u_{\alpha}(Y) A_{\alpha} X \\
\left(\nabla_{X} u_{\alpha}\right) Y=-h_{\alpha}(X, T Y)+\sum_{\beta=1}^{r}\left[u_{\beta}(Y) l_{\alpha \beta}(X)+h_{\beta}(X, Y) a_{\beta \alpha}\right] .
\end{gathered}
$$

If $N_{J}$ is the Nijenhuis tensor field of $J$, defined for any $X, Y \in \Gamma(T \bar{M})$, by

$$
N_{J}(X, Y):=[J X, J Y]+J^{2}[X, Y]-J[J X, Y]-J[X, J Y],
$$

then it verifies ([26])

$$
N_{J}(X, Y)=\left(\bar{\nabla}_{J X} J\right) Y-\left(\bar{\nabla}_{J Y} J\right) X-J\left[\left(\bar{\nabla}_{X} J\right) Y-\left(\bar{\nabla}_{Y} J\right) X\right] .
$$

Thus, we remark that in a locally metallic Riemannian manifold $(\bar{M}, \bar{g}, J)$, we have $N_{J}=0$ and the metallic structure $(\bar{g}, J)$ can be called integrable.

Now we shall define a normal $\Sigma$-metallic Riemannian structure.

Definition 4. A $\Sigma$-metallic Riemannian structure induced on a submanifold $M$ of codimension $r$ in a metallic Riemannian manifold $(\bar{M}, \bar{g}, J)$ is said to be normal if

$$
N_{T}=2 \sum_{\alpha=1}^{r} d u_{\alpha} \otimes \xi \alpha
$$

We denote by $B_{\alpha}:=T A_{\alpha}-A_{\alpha} T$ and remark that $g\left(B_{\alpha} X, Y\right)=-g\left(X, B_{\alpha} Y\right)$, for any $X, Y \in \Gamma(T M)$.

Theorem 2 ([11]). If $\left(T, g, u_{\alpha}, \xi_{\alpha},\left(a_{\alpha \beta}\right)_{r}\right)$ is the $\Sigma$-metallic Riemannian structure induced on a submanifold $M$ of codimension $r$ in a locally metallic Riemannian manifold $(\bar{M}, \bar{g}, J)$, then, for any $X, Y \in \Gamma(T M)$, we have

$$
\begin{gathered}
N_{T}(X, Y)=\sum_{\alpha=1}^{r}\left[g\left(X, \xi_{\alpha}\right) B_{\alpha} Y-g\left(Y, \xi_{\alpha}\right) B_{\alpha} X-g\left(B_{\alpha} X, Y\right) \xi_{\alpha}\right], \\
2 d u_{\alpha}(X, Y)=-g\left(B_{\alpha} X, Y\right)+\sum_{\beta=1}^{r}\left[l_{\alpha \beta}(X) g\left(Y, \xi_{\beta}\right)-l_{\alpha \beta}(Y) g\left(X, \xi_{\beta}\right)\right],
\end{gathered}
$$

where $l_{\alpha \beta}$ are the coefficients of the normal connection $\nabla^{\perp}$.

Remark 1 ([11]). In the conditions of the previous theorem, if $T$ commutes with the Weingarten operators $A_{\alpha}$, for any $\alpha \in\{1, \ldots, r\}$, i.e., $B_{\alpha}=0$, then the Nijenhuis tensor field of $T$ vanishes on $M$.

By a direct computation, for any $X, Y \in \Gamma(T M)$, we get

$$
\begin{aligned}
N_{T}(X, Y) & -2 \sum_{\alpha=1}^{r} d u_{\alpha}(X, Y) \xi_{\alpha}=\sum_{\alpha=1}^{r}\left[g\left(X, \xi_{\alpha}\right) B_{\alpha} Y-g\left(Y, \xi_{\alpha}\right) B_{\alpha} X\right] \\
& -\sum_{\alpha=1}^{r} \sum_{\beta=1}^{r}\left[l_{\alpha \beta}(X) g\left(Y, \xi_{\beta}\right)-l_{\alpha \beta}(Y) g\left(X, \xi_{\beta}\right)\right] \xi_{\alpha} .
\end{aligned}
$$


The components $N^{(1)}, N^{(2)}, N^{(3)}$ and $N^{(4)}$ of the Nijenhuis tensor field of $T$ can be computed using the similar idea from ([27]), as follows.

Remark 2 ([5]). Let $M$ be a submanifold of codimension $r$ in a locally metallic Riemannian manifold $(\bar{M}, \bar{g}, J)$ and let $\left(T, g, \eta_{\alpha}, \xi_{\alpha},\left(a_{\alpha \beta}\right)_{r}\right)$ be the $\Sigma$-metallic Riemannian structure induced on $M$. Then, for any $X, Y \in \Gamma(T M)$ and $\alpha, \beta \in\{1, \ldots, r\}$, we obtain

1. $\quad N^{(1)}(X, Y)=N_{T}(X, Y)-2 \sum_{\alpha=1}^{r} d u_{\alpha}(X, Y) \xi_{\alpha}$;

2. $N_{\alpha}^{(2)}(X, Y)=\left(\mathcal{L}_{T X} u_{\alpha}\right) Y-\left(\mathcal{L}_{T Y} u_{\alpha}\right) X$;

3. $N_{\alpha}^{(3)}(X)=\left(\mathcal{L}_{\tilde{\zeta}_{\alpha}} T\right) X$;

4. $N_{\alpha \beta}^{(4)}(X)=\left(\mathcal{L}_{\xi_{\alpha}} u_{\beta}\right) X$,

where $\mathcal{L}_{X}$ denotes the Lie derivative with respect to $X$.

We shall further provide conditions such that the induced $\Sigma$-metallic Riemannian structure to be normal.

We remark that, if the $\Sigma$-metallic Riemannian structure induced on $M$ is normal and the normal connection $\nabla^{\perp}$ of $M$ vanishes identically, i.e., $l_{\alpha \beta}=0$, then ([11])

$$
\sum_{\alpha=1}^{r} g\left(X, \xi_{\alpha}\right)\left(T A_{\alpha}-A_{\alpha} T\right)(Y)=\sum_{\alpha=1}^{r} g\left(Y, \xi_{\alpha}\right)\left(T A_{\alpha}-A_{\alpha} T\right)(X)
$$

for any $X, Y \in \Gamma(T M)$.

Theorem 3 ([11]). Let $M$ be a submanifold of codimension $r \geq 2$ in a locally metallic Riemannian manifold $(\bar{M}, \bar{g}, J)$. If the normal connection $\nabla^{\perp}$ vanishes identically and $M$ is a non-invariant submanifold with respect to the metallic structure $J$, then the vector fields $\left\{\xi_{1}, \ldots, \xi_{r}\right\}$ are linearly independent.

Theorem 4 ([11]). Let $M$ be a submanifold of codimension $r \geq 1$ in a locally metallic Riemannian manifold $(\bar{M}, \bar{g}, J)$. If the normal connection $\nabla^{\perp}$ vanishes identically and $T$ commutes with every Weingarten operator $A_{\alpha}$, then the induced $\Sigma$-metallic Riemannian structure on $M$ is normal.

Theorem 5 ([11]). Let $M$ be a submanifold of codimension $r \geq 1$ in a locally metallic Riemannian manifold $(\bar{M}, \bar{g}, J)$. If the normal connection $\nabla^{\perp}$ vanishes identically and $M$ is a non-invariant submanifold with respect to the metallic structure J, then the induced $\Sigma$-metallic Riemannian structure on $M$ is normal if and only if $T$ commutes with the Weingarten operator $A_{\alpha}$, for any $\alpha \in\{1, \ldots, r\}$.

Corollary 1 ([11]). If $M$ is a non-invariant totally umbilical (or totally geodesic) submanifold of codimension $r \geq 1$ in a locally metallic Riemannian manifold $(\bar{M}, \bar{g}, J)$ such that the normal connection $\nabla^{\perp}$ vanishes identically, then the $\Sigma$-metallic Riemannian structure induced on $M$ is normal.

We can observe that the matrix $\mathcal{A}:=\left(a_{\alpha \beta}\right)_{r}$ of the $\Sigma$-structure induced on an invariant submanifold $M$ by the metallic Riemannian structure $(\bar{g}, J)$ from $\bar{M}$ is a metallic matrix, that is a matrix which verifies

$$
\mathcal{A}^{2}=p \cdot \mathcal{A}+q \cdot I_{r}
$$

where $I_{r}$ is the identically matrix of order $r$.

If $\mathcal{A}:=\left(a_{\alpha \beta}\right)_{r}$ is a metallic matrix, then $\sum_{\gamma=1}^{r} a_{\alpha \gamma} a_{\gamma \beta}=p a_{\alpha \beta}+q \delta_{\alpha \beta}$ and we obtain $u_{\beta}\left(\xi_{\alpha}\right)=0$, which implies that $T^{2} \xi_{\alpha}=p T \xi_{\alpha}+q \xi_{\alpha}$ and $J \xi_{\alpha}=T \xi_{\alpha}$, for any $\alpha \in\{1, \ldots, r\}$. 
3.1. Invariant Submanifolds in a Metallic Riemannian Manifold $(\bar{M}, \bar{g}, J)$

A submanifold $M$ of $\bar{M}$ is called invariant if $J\left(T_{x} M\right) \subseteq T_{x} M$, for any $x \in M$. In this case, $J\left(T_{x}^{\perp} M\right) \subseteq T_{x}^{\perp} M$, for any $x \in M$.

Proposition 7 ([4]). Let $M$ be an isometrically immersed submanifold in a metallic Riemannian manifold $(\bar{M}, \bar{g}, J)$. Then $M$ is invariant if and only if $(M, g, T)$ is a metallic Riemannian manifold, whenever $T$ is non-trivial.

Proposition 8 ([4]). Let $M$ be an invariant submanifold of codimension $r$ isometrically immersed in a locally metallic Riemannian manifold $(\bar{M}, \bar{g}, J)$. Then, for any $X, Y \in \Gamma(T M)$ :

$$
\sum_{\alpha=1}^{r} h_{\alpha}(X, J Y) N_{\alpha}=\sum_{\alpha=1}^{r} h_{\alpha}(X, Y) J N_{\alpha}=\sum_{\alpha=1}^{r} h_{\alpha}(J X, Y) N_{\alpha}
$$

and for any $1 \leq \alpha \leq r$ :

$$
h_{\alpha}(J X, J Y)=p h_{\alpha}(X, J Y)+q h_{\alpha}(X, Y) .
$$

Remark 3 ([4]). Let $M$ be an invariant submanifold of codimension $r$ isometrically immersed in a locally metallic Riemannian manifold $(\bar{M}, \bar{g}, J)$ and let $\left(T, g, u_{\alpha}, \xi_{\alpha},\left(a_{\alpha \beta}\right)_{r}\right)$ be the $\Sigma$-metallic Riemannian structure induced on $M$. Then $\xi_{\alpha}$ are zero vector fields, the 1 -forms $u_{\alpha}$ vanish identically on $M$, for any $1 \leq \alpha \leq r$, and for any $X \in \Gamma(T M)$ :

$$
J X=T X, \quad J N_{\alpha}=\sum_{\beta=1}^{r} a_{\alpha \beta} N_{\beta}, \text { for any } 1 \leq \alpha \leq r .
$$

Also, the $\Sigma$-metallic Riemannian structure satisfies, for any $X, Y \in \Gamma(T M)$ and $1 \leq \alpha, \beta \leq r$ :

$$
\begin{gathered}
T^{2}=p \cdot T+q \cdot I, \\
a_{\alpha \beta}=a_{\beta \alpha}, \quad \sum_{\gamma=1}^{r} a_{\alpha \gamma} a_{\gamma \beta}=q \delta_{\alpha \beta}+p a_{\alpha \beta}, \\
X\left(a_{\alpha \beta}\right)=g\left(\left(\bar{\nabla}_{X} J\right) N_{\alpha}, N_{\beta}\right)-\sum_{\gamma=1}^{r}\left[a_{\alpha \gamma} \lambda_{\gamma \beta}(X)+a_{\beta \gamma} \lambda_{\gamma \alpha}(X)\right], \\
g(T X, Y)=g(X, T Y), \quad g(T X, T Y)=p g(X, T Y)+q g(X, Y) .
\end{gathered}
$$

Proposition 9 ([4]). If $M$ is an invariant submanifold of codimension $r$ isometrically immersed in a metallic Riemannian manifold $(\bar{M}, \bar{g}, J)$, then the $\Sigma$-metallic Riemannian structure induced on $M$ is $\left(T, g, u_{\alpha}=0, \xi_{\alpha}=0,\left(a_{\alpha \beta}\right)_{r}\right)$ and we get

$$
\begin{gathered}
\left(\left(\bar{\nabla}_{X} J\right) Y\right)^{\top}=\left(\nabla_{X} T\right) Y, \\
\left(\left(\bar{\nabla}_{X} J\right) Y\right)^{\perp}=\sum_{\alpha=1}^{r}\left[h_{\alpha}(X, T Y) N_{\alpha}-h_{\alpha}(X, Y) n N_{\alpha}\right] \\
\left(\left(\bar{\nabla}_{X} J\right) N_{\alpha}\right)^{\top}=T\left(A_{\alpha} X\right)-\sum_{\beta=1}^{r} a_{\alpha \beta} A_{\beta} X \\
\left(\left(\bar{\nabla}_{X} J\right) N_{\alpha}\right)^{\perp}=\sum_{\beta=1}^{r}\left[X\left(a_{\alpha \beta}\right)+\sum_{\gamma=1}^{r}\left(a_{\alpha \gamma} \lambda_{\gamma \beta}(X)+a_{\beta \gamma} \lambda_{\gamma \alpha}(X)\right)\right] N_{\beta},
\end{gathered}
$$

for any $X, Y \in \Gamma(T M)$ and $1 \leq \alpha \leq r$. 
Corollary 2 ([4]). If $\left(T, g, u_{\alpha}=0, \xi_{\alpha}=0,\left(a_{\alpha \beta}\right)_{r}\right)$ is the $\Sigma$-metallic Riemannian structure induced on an invariant submanifold $M$ of codimension $r$ isometrically immersed in a locally metallic Riemannian manifold $(\bar{M}, \bar{g}, J)$, then

$$
\begin{gathered}
\nabla T=0, \quad \sum_{\alpha=1}^{r}\left[h_{\alpha}(X, T Y) N_{\alpha}-h_{\alpha}(X, Y) n N_{\alpha}\right]=0, \\
T\left(A_{\alpha} X\right)-\sum_{\beta=1}^{r} a_{\alpha \beta} A_{\beta} X=0, \\
X\left(a_{\alpha \beta}\right)+\sum_{\gamma=1}^{r}\left[a_{\alpha \gamma} \lambda_{\gamma \beta}(X)+a_{\beta \gamma} \lambda_{\gamma \alpha}(X)\right]=0,
\end{gathered}
$$

for any $X, Y \in \Gamma(T M)$ and $1 \leq \alpha, \beta \leq r$.

Proposition 10 ([4]). If $\left(T, g, u_{\alpha}=0, \xi_{\alpha}=0,\left(a_{\alpha \beta}\right)_{r}\right)$ is the $\Sigma$-metallic Riemannian structure induced on an invariant submanifold $M$ of codimension $r$ isometrically immersed in a locally metallic Riemannian manifold $(\bar{M}, \bar{g}, J)$, then the Nijenhuis tensor field of $T$ vanishes identically on $M$ and $T$ commutes by $A_{\alpha}$, i.e., $T A_{\alpha}=A_{\alpha} T$, for any $1 \leq \alpha \leq r$.

\subsection{Anti-Invariant Submanifolds a Metallic Riemannian Manifold $(\bar{M}, \bar{g}, J)$}

A submanifold $M$ of $\bar{M}$ is called anti-invariant if $J\left(T_{x} M\right) \subseteq T_{x}^{\perp} M$, for any $x \in M$.

Proposition 11 ([5]). Let $M$ be an anti-invariant submanifold of codimension $r$ isometrically immersed in a locally metallic Riemannian manifold $(\bar{M}, \bar{g}, J)$. Then, for any $X, Y \in \Gamma(T M)$ :

$$
\begin{gathered}
\sum_{\alpha=1}^{r} h_{\alpha}(X, Y) t N_{\alpha}=-\sum_{\alpha=1}^{r} g\left(J Y, N_{\alpha}\right) A_{\alpha} X \\
\sum_{\alpha=1}^{r} h_{\alpha}(X, Y) n N_{\alpha}=\sum_{\alpha=1}^{r} g\left(J Y, N_{\alpha}\right) \nabla_{X}^{\frac{1}{X}} N_{\alpha}+\sum_{\alpha=1}^{r} X\left(g\left(J Y, N_{\alpha}\right)\right) N_{\alpha}-J\left(\nabla_{X} Y\right) .
\end{gathered}
$$

Remark 4 ([5]). Let $M$ be an anti-invariant submanifold of codimension $r$ isometrically immersed in a metallic Riemannian manifold $(\bar{M}, \bar{g}, J)$ and let $\left(T, g, u_{\alpha}, \xi_{\alpha},\left(a_{\alpha \beta}\right)_{r}\right)$ be the $\Sigma$-metallic Riemannian structure induced on $M$. Then $T$ vanishes identically on $M$ and, for any $X \in \Gamma(T M)$ :

$$
J X=\sum_{\alpha=1}^{r} u_{\alpha}(X) N_{\alpha}
$$

Also, the $\Sigma$-metallic Riemannian structure satisfies, for any $X \in \Gamma(T M)$ and $1 \leq \alpha, \beta \leq r$ :

$$
\begin{gathered}
\sum_{\alpha=1}^{r} u_{\alpha} \otimes \xi_{\alpha}=q \cdot I, \quad \sum_{\beta=1}^{r} a_{\alpha \beta} u_{\beta}(X)=p u_{\alpha}(X), \quad a_{\alpha \beta}=a_{\beta \alpha}, \\
u_{\beta}\left(\xi_{\alpha}\right)=q \delta_{\alpha \beta}+p a_{\alpha \beta}-\sum_{\gamma=1}^{r} a_{\alpha \gamma} a_{\gamma \beta}, \quad \sum_{\beta=1}^{r} a_{\alpha \beta} \xi_{\beta}=p \xi_{\alpha}, \quad u_{\alpha}(X)=g\left(X, \xi_{\alpha}\right), \\
X\left(a_{\alpha \beta}\right)=g\left(\left(\bar{\nabla}_{X} J\right) N_{\alpha}, N_{\beta}\right)-\left[h_{\alpha}\left(X, \xi_{\beta}\right)+h_{\beta}\left(X, \xi_{\alpha}\right)\right]-\sum_{\gamma=1}^{r}\left[a_{\alpha \gamma} \lambda_{\gamma \beta}(X)+a_{\beta \gamma} \lambda_{\gamma \alpha}(X)\right] .
\end{gathered}
$$


Proposition 12 ([5]). If $M$ is an anti-invariant submanifold of codimension $r$ isometrically immersed in a metallic Riemannian manifold $(\bar{M}, \bar{g}, J)$, then the $\Sigma$-metallic Riemannian structure induced on $M$ is $\left(T=0, g, u_{\alpha}, \xi_{\alpha},\left(a_{\alpha \beta}\right)_{r}\right)$ and we get

$$
\begin{gathered}
\left(\left(\bar{\nabla}_{X} J\right) Y\right)^{\top}=-\sum_{\alpha=1}^{r} u_{\alpha}(Y) A_{\alpha} X-\sum_{\alpha=1}^{r} h_{\alpha}(X, Y) \xi_{\alpha}, \\
\left(\left(\bar{\nabla}_{X} J\right) Y\right)^{\perp}=\sum_{\alpha=1}^{r} X\left(u_{\alpha}(Y)\right) N_{\alpha}-\sum_{1 \leq \alpha, \beta \leq r} \lambda_{\alpha \beta}(X) u_{\beta}(Y) N_{\alpha} \\
-\sum_{\alpha=1}^{r} h_{\alpha}(X, Y) n N_{\alpha}-N\left(\nabla_{X} Y\right), \\
\left(\left(\bar{\nabla}_{X} J\right) N_{\alpha}\right)^{\top}=\nabla_{X} \xi_{\alpha}-\sum_{\beta=1}^{r} a_{\alpha \beta} A_{\beta} X-\sum_{\beta=1}^{r} \lambda_{\alpha \beta}(X) \xi_{\beta}, \\
\left(\left(\bar{\nabla}_{X} J\right) N_{\alpha}\right)^{\perp}=\sum_{\beta=1}^{r}\left[X\left(a_{\alpha \beta}\right)+h_{\beta}\left(X, \xi_{\alpha}\right)\right. \\
\left.+\sum_{\gamma=1}^{r}\left(a_{\alpha \gamma} \lambda_{\gamma \beta}(X)+a_{\beta \gamma} \lambda_{\gamma \alpha}(X)\right)\right] N_{\beta}+N\left(A_{\alpha} X\right),
\end{gathered}
$$

for any $X, Y \in \Gamma(T M)$ and $1 \leq \alpha \leq r$.

Corollary 3 ([5]). If $\left(T=0, g, u_{\alpha}, \xi_{\alpha},\left(a_{\alpha \beta}\right)_{r}\right)$ is the $\Sigma$-metallic Riemannian structure induced on an anti-invariant submanifold $M$ of codimension $r$ isometrically immersed in a locally metallic Riemannian manifold $(\bar{M}, \bar{g}, J)$, then

$$
\begin{gathered}
\sum_{\alpha=1}^{r} u_{\alpha}(Y) A_{\alpha} X+\sum_{\alpha=1}^{r} h_{\alpha}(X, Y) \xi_{\alpha}=0 \\
\sum_{\alpha=1}^{r} X\left(u_{\alpha}(Y)\right) N_{\alpha}=-\sum_{1 \leq \alpha, \beta \leq r} \lambda_{\alpha \beta}(X) u_{\beta}(Y) N_{\alpha}-\sum_{\alpha=1}^{r} h_{\alpha}(X, Y) n N_{\alpha}-N\left(\nabla_{X} Y\right)=0, \\
\nabla_{X} \xi_{\alpha}-\sum_{\beta=1}^{r} a_{\alpha \beta} A_{\beta} X-\sum_{\beta=1}^{r} \lambda_{\alpha \beta}(X) \xi_{\beta}=0 \\
X\left(a_{\alpha \beta}\right)+h_{\alpha}\left(X, \xi_{\beta}\right)+h_{\beta}\left(X, \xi_{\alpha}\right)+\sum_{\gamma=1}^{r}\left[a_{\alpha \gamma} \lambda_{\gamma \beta}(X)+a_{\beta \gamma} \lambda_{\gamma \alpha}(X)\right]=0, \\
\sum_{\beta=1}^{r} h_{\alpha}\left(X, \xi_{\beta}\right) N_{\beta}=N\left(A_{\alpha} X\right)
\end{gathered}
$$

for any $X, Y \in \Gamma(T M)$ and $1 \leq \alpha \leq r$.

Proposition 13 ([5]). If $\left(T=0, g, u_{\alpha}, \xi_{\alpha},\left(a_{\alpha \beta}\right)_{r}\right)$ is the $\Sigma$-metallic Riemannian structure induced on an anti-invariant submanifold $M$ of codimension $r$ isometrically immersed in a locally metallic Riemannian manifold $(\bar{M}, \bar{g}, J)$, then $N^{(2)}$ and $N^{(3)}$ vanish identically on $M$. Moreover, if $\xi_{\alpha}$ are parallel with respect to a symmetric linear connection, for any $1 \leq \alpha \leq r$, then $N^{(1)}$ and $N^{(4)}$ vanish, too, on $M$.

\section{Slant Submanifolds in Metallic Riemannian Manifolds}

Let $M$ be a submanifold of codimension $r \in N^{*}$ isometrically immersed in a metallic Riemannian manifold $(\bar{M}, \bar{g}, J)$. For any $X \in \Gamma(T M)$, one obtains (from the CauchySchwartz inequality)

$$
\bar{g}(J X, T X) \leq\|J X\| \cdot\|T X\| .
$$


Therefore, we can consider a function $\theta: \Gamma(T M) \rightarrow\left[0, \frac{\pi}{2}\right]$, such that:

$$
\bar{g}\left(J X_{x}, T X_{x}\right)=\cos \theta\left(X_{x}\right) \cdot\left\|J X_{x}\right\| \cdot\left\|T X_{x}\right\|,
$$

for any $x \in M$ and any nonzero $X_{x} \in T_{x} M$. If $J X_{x} \neq 0$, then the angle $\theta\left(X_{x}\right)$ between $J X_{x}$ and $T_{x} M$ is called the Wirtinger angle of $X$.

Definition 5 ([5]). A slant submanifold $M$ in a metallic Riemannian manifold $(\bar{M}, \bar{g}, J)$ is a submanifold having the angle $\theta\left(X_{x}\right)=$ constant (where $\theta\left(X_{x}\right)$ is the angle between $J X_{x}$ and $T_{x} M$, for any $x \in M$ and $X_{x} \in T_{x} M$, whenever $J X_{x} \neq 0$ ). Then $\theta=: \theta\left(X_{x}\right)$ (called the slant angle of $M$ in $\bar{M}$ ) has the property

$$
\cos \theta=\frac{\|T X\|}{\|J X\|} .
$$

In this case, $i: M \rightarrow \bar{M}$ is called the slant immersion of $M$ in $\bar{M}$.

Remark 5. For $\theta=0$ (or $\theta=\frac{\pi}{2}$ ) we obtain the particular cases of invariant (or respectively, anti-invariant) submanifolds $M$ in a metallic Riemannian manifold $(\bar{M}, \bar{g}, J)$. A proper slant submanifold is a slant submanifold which is neither invariant nor anti-invariant and $i: M \rightarrow \bar{M}$ is called a proper slant immersion.

Proposition 14 ([5]). If $M$ is a slant submanifold with the slant angle $\theta$, isometrically immersed in a metallic Riemannian manifold $(\bar{M}, \bar{g}, J)$, then, for any $X, Y \in \Gamma(T M)$ :

$$
\begin{aligned}
& \bar{g}(T X, T Y)=\cos ^{2} \theta \cdot \bar{g}(X, p \cdot T Y+q \cdot Y), \\
& \bar{g}(N X, N Y)=\sin ^{2} \theta \cdot \bar{g}(X, p \cdot T Y+q \cdot Y) .
\end{aligned}
$$

We also have

$$
T^{2}=\cos ^{2} \theta \cdot(p \cdot T+q \cdot I), \quad \nabla T^{2}=p \cos ^{2} \theta \cdot \nabla T,
$$

where I is the identity on $\Gamma(T M)$.

Remark 6. From ([5]) we obtain

$$
t N=\sin ^{2} \theta \cdot(p \cdot T+q \cdot I)=\sum_{\alpha=1}^{r} u_{\alpha} \otimes \xi_{\alpha} .
$$

Like in the Riemannian product case ([28]), we can define a slant distribution in a metallic Riemannian manifold.

Definition 6 ([5]). If $M$ is an immersed submanifold in a metallic Riemannian manifold $(\bar{M}, \bar{g}, J)$, then a differentiable distribution $D$ on $M$ is called a slant distribution if the angle $\theta_{D}$ between $J X_{x}$ and the vector subspace $D_{x}$ is constant, for any $x \in M$ and any nonzero $X_{x} \in D_{x}$. The constant angle $\theta_{D}$ is called the slant angle of the distribution $D$.

Proposition 15 ([6]). If $D$ is a differentiable distribution on a submanifold $M$ of a metallic Riemannian manifold $(\bar{M}, \bar{g}, J)$ and $P_{D}$ is the orthogonal projection on $D$, then $D$ is a slant distribution if and only if there exists a constant $\lambda \in[0,1]$ such that:

$$
\left(P_{D} T\right)^{2} X=\lambda \cdot\left(p \cdot P_{D} T X+q \cdot X\right),
$$

for any $X \in \Gamma(D)$. Moreover, the slant angle $\theta_{D}$ of $D$ satisfies $\lambda=\cos ^{2} \theta_{D}$.

\section{Bi-Slant Submanifolds in Metallic Riemannian Manifolds}

The differential geometry of slant submanifolds has shown an increasing development in the early 1990's when B.-Y. Chen defined slant submanifolds in complex manifolds ([29]). 
Particular cases of bi-slant submanifolds, such as semi-invariant submanifolds in locally product Riemannian manifolds were studied in ([30,31]), semi-slant submanifolds were studied by J. L. Cabrerizo and A. Carriazo ([32-34]). Moreover, slant and semi-slant submanifolds in almost product Riemannian manifolds were studied in $([28,30])$. The hemi-slant submanifolds (called, also, pseudo-slant submanifolds) in locally decomposable Riemannian manifolds were studied by M. Atçeken et al. ([35]) and in locally product Riemannian manifolds were studied by H. M. Taştan and F. Ozdem in ([36]).

Definition 7 ([10]). If $M$ is an immersed submanifold in a metallic Riemannian manifold $(\bar{M}, \bar{g}, J)$, then $M$ is called a bi-slant submanifold if there exist two orthogonal differentiable distributions $D_{1}$ and $D_{2}$ on $M$ such that:

1. $\quad T M$ admits the orthogonal decomposition $T M=D_{1} \oplus D_{2}$;

2. $J\left(D_{1}\right) \perp D_{2}$ and $J\left(D_{2}\right) \perp D_{1}$;

3. the distributions $D_{1}$ and $D_{2}$ are slant with angles $\theta_{1} \neq \theta_{2}$.

If $M$ is a bi-slant submanifold of $(\bar{M}, \bar{g}, J)$, then $T\left(D_{1}\right) \subseteq D_{1}$ and $T\left(D_{2}\right) \subseteq D_{2}$.

We provide an example of a bi-slant submanifold in a metallic Riemannian manifold.

Example 1. We consider the Euclidean space $R^{4}$ endowed with the Euclidean metric $\langle\cdot, \cdot\rangle$ and the immersion $i: M \rightarrow R^{4}$, given by

$$
i(\alpha, \beta):=\left(\alpha \cos t, \frac{\sigma}{\sqrt{q}} \alpha \sin t, \beta, \beta\right),
$$

where $M:=\{(\alpha, \beta) \mid \alpha, \beta>0\}, t \in\left[0, \frac{\pi}{2}\right]$ and $\sigma:=\sigma_{p, q}=\frac{p+\sqrt{p^{2}+4 q}}{2}$ is a metallic number.

The local orthogonal frame on TM is

$$
Z_{1}=\cos t \frac{\partial}{\partial x_{1}}+\frac{\sigma}{\sqrt{q}} \sin t \frac{\partial}{\partial x_{2}}, Z_{2}=\frac{\partial}{\partial x_{3}}+\frac{\partial}{\partial x_{4}} .
$$

The structure $J: R^{4} \rightarrow R^{4}$ given by

$$
J\left(\frac{\partial}{\partial x_{k}}\right):= \begin{cases}\sigma \frac{\partial}{\partial x_{k}}, & k \in\{1,3\} \\ \bar{\sigma} \frac{\partial}{\partial x_{k}}, & k \in\{2,4\}\end{cases}
$$

is a metallic structure, where $\bar{\sigma}:=p-\sigma$. Since

$$
J Z_{1}=\sigma \cos t \frac{\partial}{\partial x_{1}}-\sqrt{q} \sin t \frac{\partial}{\partial x_{2}}, \quad J Z_{2}=\sigma \frac{\partial}{\partial x_{3}}+\bar{\sigma} \frac{\partial}{\partial x_{4}},
$$

we remark that $\left\langle J Z_{1}, Z_{1}\right\rangle=\sigma \cos 2 t,\left\langle J Z_{2}, Z_{2}\right\rangle=\sigma+\bar{\sigma}$ and

$$
\begin{gathered}
\left\|Z_{1}\right\|^{2}=\frac{p \sigma}{q} \sin ^{2} t+1, \quad\left\|Z_{2}\right\|^{2}=2, \\
\left\|J Z_{1}\right\|^{2}=\sigma\left(\sigma-p \sin ^{2} t\right), \quad\left\|J Z_{2}\right\|^{2}=p^{2}+2 q .
\end{gathered}
$$

If $D_{1}:=\operatorname{span}\left\{Z_{1}\right\}$ and $D_{2}:=\operatorname{span}\left\{Z_{2}\right\}$, then $\cos \theta_{1}=\frac{\sqrt{q} \cos 2 t}{\sqrt{\left(\sigma-p \cos ^{2} t\right)\left(\sigma-p \sin ^{2} t\right)}}$ and $\cos \theta_{2}=\frac{p}{\sqrt{2\left(p^{2}+2 q\right)}}$. Thus, $D_{1}$ and $D_{2}$ are the slant distributions, with the slant angles $\theta_{1}$ and $\theta_{2}$, respectively. 
Let $M_{\theta_{1}}$ and $M_{\theta_{2}}$ be the integral manifolds of the distributions $D_{1}$ and $D_{2}$, respectively. Thus, $M_{\theta_{1}} \times M_{\theta_{2}}$ is a bi-slant submanifold in the metallic Riemannian manifold $\left(R^{4},\langle\cdot, \cdot\rangle, J\right)$, with the metric

$$
g:=\left(\frac{p \sigma}{q} \sin ^{2} t+1\right) g_{1}+2 g_{2} .
$$

\subsection{Semi-Slant Submanifolds in Metallic Riemannian Manifolds}

Semi-slant submanifolds in a metallic Riemannian manifold are particular cases of bi-slant submanifolds, which can be defined in a similar manner as semi-slant submanifolds in a locally product Riemannian manifold ([28]).

Definition 8 ([6]). A semi-slant submanifold $M$ in a metallic Riemannian manifold $(\bar{M}, \bar{g}, J)$ is a submanifold which has two orthogonal differentiable distributions $D$ and $D^{\theta}$, such that:

1. $T M$ admits the orthogonal decomposition $T M=D \oplus D^{\theta}$;

2. the distribution $D$ is invariant;

3. the distribution $D^{\theta}$ is slant with angle $\theta \neq 0$, and $J\left(D^{\theta}\right) \perp D$.

Moreover, $M$ is called a proper semi-slant submanifold if $\theta \in\left(0, \frac{\pi}{2}\right)$.

Remark 7. Let $M$ be a semi-slant submanifold in a metallic Riemannian manifold $(\bar{M}, \bar{g}, J)$ with $T M=D \oplus D^{\theta}$. If $\theta=\frac{\pi}{2}$, then $M$ is a semi-invariant submanifold of $\bar{M}$.

Let $P_{1}$ and $P_{2}$ be the orthogonal projections on $D$ and $D^{\theta}$. The decomposition of $X \in \Gamma(T M)$ is given by $X=P_{1} X+P_{2} X$, where $P_{1} X \in \Gamma(D)$ and $P_{2} X \in \Gamma\left(D^{\theta}\right)$.

Proposition 16 ([6]). Let $M$ be a semi-slant submanifold in a metallic Riemannian manifold $(\bar{M}, \bar{g}, J)$. Then, for any $X \in \Gamma(T M)$ :

$$
\begin{gathered}
J X=T P_{1} X+T P_{2} X+N P_{2} X=P_{1} T X+P_{2} T X+N P_{2} X, \\
J P_{1} X=T P_{1} X, \quad N P_{1} X=0, \quad T P_{2} X \in \Gamma\left(D^{\theta}\right) .
\end{gathered}
$$

Remark 8 ([6]). Let $M$ be a semi-slant submanifold in a metallic Riemannian manifold $(\bar{M}, \bar{g}, J)$. Then, for any $X \in \Gamma(T M)$, we get

$$
\bar{g}\left(J P_{2} X, T P_{2} X\right)=\cos \theta(X) \cdot\left\|T P_{2} X\right\| \cdot\left\|J P_{2} X\right\|
$$

and for $P_{2} X \neq 0$, the angle $\theta$ of the distribution $D^{\theta}$ is constant, given by

$$
\cos \theta(X)=\frac{\left\|T P_{2} X\right\|}{\left\|J P_{2} X\right\|} .
$$

Proposition 17 ([6]). If $M$ is a semi-slant submanifold with the slant angle $\theta$, isometrically immersed in a metallic Riemannian manifold $(\bar{M}, \bar{g}, J)$, then, for any $X, Y \in \Gamma(T M)$ :

$$
\begin{gathered}
\bar{g}\left(T P_{2} X, T P_{2} Y\right)=\cos ^{2} \theta \cdot \bar{g}\left(p \cdot T P_{2} X+q \cdot P_{2} X, P_{2} Y\right) \\
\bar{g}(N X, N Y)=\sin ^{2} \theta \cdot \bar{g}\left(p \cdot T P_{2} X+q \cdot P_{2} X, P_{2} Y\right) .
\end{gathered}
$$

We also have

$$
\left(T P_{2}\right)^{2}=\cos ^{2} \theta \cdot\left(p \cdot T P_{2}+q \cdot I\right), \quad \nabla\left(T P_{2}\right)^{2}=p \cos ^{2} \theta \cdot \nabla\left(T P_{2}\right),
$$

where I is the identity on $\Gamma\left(D^{\theta}\right)$. 
Proposition 18 ([6]). A necessary and sufficient condition for a submanifold $M$ in a metallic Riemannian manifold $(\bar{M}, \bar{g}, J)$ to be a semi-slant submanifold in $\bar{M}$ is to exist a constant $\lambda \in[0,1)$, such that

$$
D_{0}=\left\{X \in \Gamma(T M) \mid T^{2} X=\lambda(p \cdot T X+q \cdot X)\right\}
$$

is a differentiable distribution, and $N X=0$, for any $X \in \Gamma(T M)$ orthogonal to $D_{0}$.

Proposition 19 ([6]). Let $M$ be a semi-slant submanifold in a locally metallic Riemannian manifold $(\bar{M}, \bar{g}, J)$. Then:

(i) a necessary and sufficient condition for the integrability of the distribution $D$ is:

$$
h(X, T Y)=h(T X, Y) \text { or } J A_{V} X=A_{V} J X,
$$

for any $X, Y \in \Gamma(D)$ and $V \in \Gamma\left(T^{\perp} M\right)$;

(ii) a necessary and sufficient condition for the integrability of the distribution $D^{\theta}$ is:

$$
P_{1}\left(\nabla_{Z} T W-\nabla_{W} T Z\right)=P_{1}\left(A_{N W} Z-A_{N Z} W\right),
$$

for any $Z, W \in \Gamma\left(D^{\theta}\right)$.

Remark 9 ([6]). The condition $\nabla T=0$ implies the integrability of the distributions $D$ and $D^{\theta}$ on a semi-slant submanifold $M$ in a locally metallic Riemannian manifold $(\bar{M}, \bar{g}, J)$.

Proposition 20 ([6]). If $M$ is a semi-slant submanifold in a locally metallic Riemannian manifold $(\bar{M}, \bar{g}, J)$, then $\nabla N=0$ if and only if, for any $X \in \Gamma(T M)$ and $V \in \Gamma\left(T^{\perp} M\right)$ :

$$
A_{n V} X=T A_{V} X=A_{V} T X .
$$

Definition 9. Let $M$ be a semi-slant submanifold in a metallic Riemannian manifold $(\bar{M}, \bar{g}, J)$. We say that $M$ is a $D-D^{\theta}$ mixed totally geodesic if $h(X, Z)=0$, for any $X \in \Gamma(D)$ and any $Z \in \Gamma\left(D^{\theta}\right)$.

Proposition 21 ([6]). A necessary and sufficient condition for the semi-slant submanifold $M$ in a locally metallic Riemannian manifold $(\bar{M}, \bar{g}, J)$ to be a $D-D^{\theta}$ mixed totally geodesic submanifold is $A_{V} X \in \Gamma(D)$ and $A_{V} Z \in \Gamma\left(D^{\theta}\right)$, for any $X \in \Gamma(D), Z \in \Gamma\left(D^{\theta}\right)$ and $V \in \Gamma\left(T^{\perp} M\right)$.

Proposition 22 ([6]). If $M$ is a proper semi-slant submanifold in a locally metallic Riemannian manifold $(\bar{M}, \bar{g}, J)$, then $M$ is a $D-D^{\theta}$ mixed totally geodesic submanifold if one of the following conditions are true, for any $X \in \Gamma(D), Z \in \Gamma\left(D^{\theta}\right)$ and $V \in \Gamma\left(T^{\perp} M\right)$ :

(i) $\left(\bar{\nabla}_{X} N\right) Z=0$ and $h(X, Z)$ is not an eigenvector of the tensor field $n$ with the eigenvalue $-\frac{q}{p}$;

(ii) $A_{n V} X=T A_{V} X=A_{V} T X$ and $h(X, Z)$ is not an eigenvector of the tensor field $n$.

Proposition 23 ([6]). If $M$ is a $D-D^{\theta}$ mixed totally geodesic proper semi-slant submanifold in a locally metallic Riemannian manifold $(\bar{M}, \bar{g}, J)$, then $\left(\bar{\nabla}_{X} N\right) Y=0$, for any $X \in \Gamma(D)$ and $Y \in \Gamma\left(D^{\theta}\right)$.

Example 2. For $t=0$ in the Example 1, we obtain $\theta_{1}=0$ and the submanifold $M_{0} \times M_{\theta_{2}}$ is a semi-slant submanifold in the metallic Riemannian manifold $\left(R^{4},\langle\cdot, \cdot\rangle, J\right)$.

\subsection{Hemi-Slant Submanifolds in Metallic Riemannian Manifolds}

Hemi-slant submanifolds in a metallic Riemannian manifold are particular cases of bislant submanifolds, which can be defined in a similar manner as hemi-slant submanifolds in a locally product Riemannian manifold ([36]). 
Definition 10 ([7]). A hemi-slant submanifold $M$ in a metallic Riemannian manifold $(\bar{M}, \bar{g}, J)$ is a submanifold which has two orthogonal differentiable distributions $D^{\theta}$ and $D^{\perp}$, such that:

1. $T M$ admits the orthogonal decomposition $T M=D^{\theta} \oplus D^{\perp}$;

2. the distribution $D^{\perp}$ is anti-invariant;

3. the distribution $D^{\theta}$ is slant with angle $\theta \neq \frac{\pi}{2}$, and $J\left(D^{\theta}\right) \perp D^{\perp}$.

Moreover, $M$ is called a proper hemi-slant submanifold if $\theta \in\left(0, \frac{\pi}{2}\right)$.

Remark 10. Let $M$ be a hemi-slant submanifold in a metallic Riemannian manifold $(\bar{M}, \bar{g}, J)$ with $T M=D^{\theta} \oplus D^{\perp}$. If $\theta=0$, then $M$ is a semi-invariant submanifold of $\bar{M}$.

Let $P_{1}$ and $P_{2}$ be the orthogonal projections on $D^{\theta}$ and $D^{\perp}$. The decomposition of $X \in \Gamma(T M)$ is given by $X=P_{1} X+P_{2} X$, where $P_{1} X \in \Gamma\left(D^{\theta}\right)$ and $P_{2} X \in \Gamma\left(D^{\perp}\right)$.

Proposition 24 ([7]). Let $M$ be a hemi-slant submanifold in a metallic Riemannian manifold $(\bar{M}, \bar{g}, J)$. Then, for any $X \in \Gamma(T M)$ :

$$
\begin{aligned}
& J X=T P_{1} X+N P_{1} X+N P_{2} X=T P_{1} X+N X \\
& J P_{2} X=N P_{2} X, \quad T P_{2} X=0, \quad T P_{1} X \in \Gamma\left(D^{\theta}\right) .
\end{aligned}
$$

Remark 11 ([7]). Let $M$ be a hemi-slant submanifold in a metallic Riemannian manifold $(\bar{M}, \bar{g}, J)$. Then, for any $X \in \Gamma(T M)$, we get

$$
\bar{g}\left(J P_{1} X, T P_{1} X\right)=\cos \theta(X) \cdot\left\|T P_{1} X\right\| \cdot\left\|J P_{1} X\right\|
$$

and for $P_{1} X \neq 0$, the angle $\theta$ of the distribution $D^{\theta}$ is constant, given by

$$
\cos \theta(X)=\frac{\left\|T P_{1} X\right\|}{\left\|J P_{1} X\right\|} .
$$

Proposition 25 ([7]). If $M$ is a hemi-slant submanifold with the slant angle $\theta$, isometrically immersed in a metallic Riemannian manifold $(\bar{M}, \bar{g}, J)$, then, for any $X, Y \in \Gamma(T M)$ :

$$
\begin{gathered}
\bar{g}\left(T P_{1} X, T P_{1} Y\right)=\cos ^{2} \theta \cdot \bar{g}\left(p \cdot T P_{1} X+q \cdot P_{1} X, P_{1} Y\right) \\
\bar{g}(N X, N Y)=\sin ^{2} \theta \cdot \bar{g}\left(p \cdot T P_{1} X+q \cdot P_{1} X, P_{1} Y\right) .
\end{gathered}
$$

We also have

$$
\left(T P_{1}\right)^{2}=\cos ^{2} \theta \cdot\left(p \cdot T P_{1}+q \cdot I\right), \quad \nabla\left(T P_{1}\right)^{2}=p \cos ^{2} \theta \cdot \nabla\left(T P_{1}\right),
$$

where I is the identity on $\Gamma\left(D^{\theta}\right)$.

Definition 11. Let $M$ be a hemi-slant submanifold in a metallic Riemannian manifold $(\bar{M}, \bar{g}, J)$. We say that $M$ is a $D^{\theta}-D^{\perp}$ mixed totally geodesic if $h(X, Z)=0$, for any $X \in \Gamma\left(D^{\theta}\right)$ and any $Z \in \Gamma\left(D^{\perp}\right)$.

Proposition 26 ([7]). A necessary and sufficient condition for a submanifold $M$ in a metallic Riemannian manifold $(\bar{M}, \bar{g}, J)$ to be a hemi-slant submanifold in $\bar{M}$ is to exist a constant $\lambda \in[0,1)$, such that

$$
D_{0}=\left\{X \in \Gamma(T M) \mid T^{2} X=\lambda(p \cdot T X+q \cdot X)\right\}
$$

is a differentiable distribution, and $T X=0$, for any $X \in \Gamma(T M)$ orthogonal to $D_{0}$. 
Proposition 27 ([7]). Let $M$ be a hemi-slant submanifold in a locally metallic Riemannian manifold $(\bar{M}, \bar{g}, J)$. Then:

(i) the distribution $D^{\theta}$ is integrable and, for any $X, Y \in \Gamma\left(D^{\theta}\right)$, we get

$$
\nabla_{X} T Y-\nabla_{Y} T X-A_{N Y} X+A_{N X} Y \in \Gamma\left(D^{\theta}\right)
$$

(ii) a necessary and sufficient condition for the integrability of the distribution $D^{\perp}$ is:

$$
A_{N Z} W=0 \text { or }\left(\nabla_{Z} T\right) W=\left(\nabla_{W} T\right) Z,
$$

for any $Z, W \in \Gamma\left(D^{\perp}\right)$.

Remark 12 ([7]). The condition $\left(\nabla_{Z} T\right) W=0$, for any $Z, W \in \Gamma\left(D^{\perp}\right)$, implies the integrability of the distribution $D^{\perp}$ on a semi-slant submanifold $M$ in a locally metallic Riemannian manifold $(\bar{M}, \bar{g}, J)$.

Proposition 28 ([7]). If $M$ is a hemi-slant submanifold in a locally metallic Riemannian manifold $(\bar{M}, \bar{g}, J)$, then $\nabla N=0$ if and only if, for any $X \in \Gamma(T M)$ and $V \in \Gamma\left(T^{\perp} M\right)$ :

$$
A_{n V} X=T A_{V} X=A_{V} T X .
$$

Proposition 29 ([7]). A necessary and sufficient condition for the hemi-slant submanifold $M$ in a locally metallic Riemannian manifold $(\bar{M}, \bar{g}, J)$ to be a $D^{\theta}-D^{\perp}$ mixed totally geodesic submanifold is $A_{V} X \in \Gamma\left(D^{\theta}\right)$ and $A_{V} Y \in \Gamma\left(D^{\perp}\right)$, for any $X \in \Gamma\left(D^{\theta}\right), Y \in \Gamma\left(D^{\perp}\right)$ and $V \in \Gamma\left(T^{\perp} M\right)$.

Proposition 30 ([7]). If $M$ is a proper hemi-slant submanifold in a locally metallic Riemannian manifold $(\bar{M}, \bar{g}, J)$ and $\left(\bar{\nabla}_{X} N\right) Y=0$, for any $X, Y \in \Gamma(T M)$, then $M$ is a $D^{\theta}-D^{\perp}$ mixed totally geodesic submanifold in $\bar{M}$.

Example 3. For $t=\frac{\pi}{4}$ in the Example 1, we obtain $\theta_{1}=\frac{\pi}{2}$ and the submanifold $M_{\frac{\pi}{2}} \times M_{\theta_{2}}$ is a hemi-slant submanifold in the metallic Riemannian manifold $\left(R^{4},\langle\cdot, \cdot\rangle, J\right)$.

\section{Warped Product Bi-Slant Submanifolds in Metallic Riemannian Manifolds}

Many properties for warped product manifolds and submanifolds were presented by B.-Y. Chen in his book ([37]). Warped product submanifolds in locally Riemannian product manifolds were studied by F. R. Al-Solamy and S. Uddin ([38-40]), B. Şahin ([41]), M. Atçeken ([42-44]).

In this section we present some results regarding the existence and nonexistence of non-trivial semi-invariant, semi-slant and hemi-slant warped product submanifolds in locally metallic Riemannian manifolds and we provide examples.

Let $\left(M_{1}, g_{1}\right)$ and $\left(M_{2}, g_{2}\right)$ be two Riemannian manifolds and denote by $\pi_{1}$ and $\pi_{2}$ the projection maps from the product manifold $M_{1} \times M_{2}$ onto $M_{1}$ and $M_{2}$, respectively.

Definition 12 ([45]). If $g$ is the Riemannian metric on $M_{1} \times M_{2}$ defined by

$$
g:=\pi_{1}^{*} g_{1}+\left(f \circ \pi_{1}\right)^{2} \pi_{2}^{*} g_{2},
$$

where $f: M_{1} \rightarrow(0, \infty)$ is a differentiable function on $M_{1}$, then $M_{1} \times{ }_{f} M_{2}=:\left(M_{1} \times M_{2}, g\right)$ is called the warped product Riemannian manifold of $M_{1}$ and $M_{2}$, having the warping function $f$. Moreover, $M_{1} \times_{f} M_{2}$ is called trivial if $f$ is constant. In this case, it is just a Riemannian product $M_{1} \times M_{2}$, where $M_{2}$ is equipped with the metric $f^{2} g_{2}$ (which is homothetic to $g_{2}$ ).

For simplification, we will use $\left(f \circ \pi_{1}\right)^{2}=: f^{2}, \pi_{1}^{*} g_{1}=: g_{1}$ and $\pi_{2}^{*} g_{2}=: g_{2}$. 
It is known that $M_{1}$ is a totally geodesic and $M_{2}$ is a totally umbilical submanifold of the warped product manifold $M_{1} \times{ }_{f} M_{2}$ ([45]).

Definition 13. If $M_{1}$ and $M_{2}$ are slant submanifolds in a metallic Riemannian manifold $(\bar{M}, \bar{g}, J)$, then the warped product $M_{1} \times_{f} M_{2}$ is called warped product bi-slant submanifold.

Moreover, $M_{1} \times_{f} M_{2}$ is called a proper warped product bi-slant submanifold if $\theta_{1} \neq \theta_{2}$.

Definition 14. Let $M_{1} \times{ }_{f} M_{2}$ be a warped product submanifold in a metallic Riemannian manifold $(\bar{M}, \bar{g}, J)$. If one of the components $M_{i}(i \in\{1,2\})$ is invariant (respectively, anti-invariant) submanifold in $\bar{M}$ and the other one is slant having the slant angle $\theta \in\left(0, \frac{\pi}{2}\right]$ (or $\theta \in\left[0, \frac{\pi}{2}\right)$, respectively), then the submanifold $M$ is called a warped product semi-slant (respectively, warped product hemi-slant) submanifold.

Definition 15. Let $M_{1} \times_{f} M_{2}$ be a warped product semi-slant (respectively, hemi-slant) submanifold in a metallic Riemannian manifold $(\bar{M}, \bar{g}, J)$ such that the slant angle is $\theta=\frac{\pi}{2}$ (respectively, $\theta=0$ ). Then the warped products $M_{\top} \times_{f} M_{\perp}$ and $M_{\perp} \times_{f} M_{\top}$ are called warped product semi-invariant submanifolds.

Proposition 31 ([10]). If $M_{1} \times_{f} M_{2}$ is a warped product bi-slant submanifold in a locally metallic Riemannian manifold $(\bar{M}, \bar{g}, J)$, then

$$
\begin{gathered}
\bar{g}(h(X, Y), N Z)=-\bar{g}(h(X, Z), N Y), \quad \bar{g}(h(X, Z), N W)=0, \\
\bar{g}(h(Z, W), N X)=T X(\ln f) \bar{g}(Z, W)-X(\ln f) \bar{g}(Z, T W),
\end{gathered}
$$

for any $X, Y \in \Gamma\left(T M_{1}\right)$ and $Z, W \in \Gamma\left(T M_{2}\right)$.

Proposition 32 ([10]). If $M_{\top} \times_{f} M_{\perp}$ is a warped product semi-invariant submanifold in a locally metallic Riemannian manifold $(\bar{M}, \bar{g}, J)$, then it is a trivial warped product Riemannian manifold.

Proposition 33 ([10]). If $M_{\perp} \times_{f} M_{\top}$ is a warped product semi-invariant submanifold in a locally metallic Riemannian manifold $(\bar{M}, \bar{g}, J)$, then it is a trivial warped product Riemannian manifold if and only if

$$
t \nabla \frac{1}{Z} J X=-(T-p I) A_{J X} Z,
$$

for any $X \in \Gamma\left(T M_{\perp}\right)$ and $Z \in \Gamma\left(T M_{\top}\right)$.

Proposition 34 ([10]). If $M_{1 \top} \times_{f} M_{2 \top}$ is a warped product submanifold in a locally metallic Riemannian manifold $(\bar{M}, \bar{g}, J)$ and $X(\ln f) \neq 0$, for any $X \in \Gamma\left(T M_{1 \top}\right)$, then

$$
T X(\ln f)=\widetilde{\sigma} X(\ln f),
$$

for any $X \in \Gamma\left(T M_{1 \top}\right)$ and $Z \in \Gamma\left(T M_{2 \top}\right)$, where $\widetilde{\sigma} \in\{\sigma, \bar{\sigma}\}, \sigma:=\sigma_{p, q}$ is a metallic number and $\bar{\sigma}:=p-\sigma$.

Proposition 35 ([10]). If $M_{1 \perp} \times_{f} M_{2 \perp}$ is a warped product submanifold in a locally metallic Riemannian manifold $(\bar{M}, \bar{g}, J)$, then it is a trivial warped product Riemannian manifold if and only if

$$
t \nabla \frac{\perp}{Z} N X=p t h(X, Z),
$$

for any $X \in \Gamma\left(T M_{1 \perp}\right)$ and $Z \in \Gamma\left(T M_{2 \perp}\right)$.

We provide an example of a non-trivial warped product semi-invariant submanifold in a metallic Riemannian manifold. 
Example 4. We consider the Euclidean space $R^{5}$ endowed with the Euclidean metric $\langle\cdot, \cdot\rangle$ and the immersion $i: M \rightarrow R^{5}$, given by

$$
i(f, \alpha, \beta):=\left(f \sin \alpha, f \cos \alpha, f \sin \beta, f \cos \beta, \sqrt{\frac{p \sigma}{q}} f\right),
$$

where $M:=\left\{(f, \alpha, \beta) \mid f>0, \alpha, \beta \in\left(0, \frac{\pi}{2}\right)\right\}$ and $\sigma:=\sigma_{p, q}$ is a metallic number.

The local orthogonal frame on $T M$ is

$$
\begin{aligned}
& Z_{1}=\sin \alpha \frac{\partial}{\partial x_{1}}+\cos \alpha \frac{\partial}{\partial x_{2}}+\sin \beta \frac{\partial}{\partial x_{3}}+\cos \beta \frac{\partial}{\partial x_{4}}+\sqrt{\frac{p \sigma}{q} \frac{\partial}{\partial x_{5}}} \\
& Z_{2}=f \cos \alpha \frac{\partial}{\partial x_{1}}-f \sin \alpha \frac{\partial}{\partial x_{2}}, Z_{3}=f \cos \beta \frac{\partial}{\partial x_{3}}-f \sin \beta \frac{\partial}{\partial x_{4}} .
\end{aligned}
$$

The structure $J: R^{5} \rightarrow R^{5}$ given by

$$
J\left(\frac{\partial}{\partial x_{k}}\right):= \begin{cases}\sigma \frac{\partial}{\partial x_{k}}, & k \in\{1,2\} \\ \bar{\sigma} \frac{\partial}{\partial x_{k}}, & k \in\{3,4,5\}\end{cases}
$$

is a metallic structure, where $\bar{\sigma}:=1-\sigma$. Since

$$
\begin{gathered}
J Z_{1}=\sigma \sin \alpha \frac{\partial}{\partial x_{1}}+\sigma \cos \alpha \frac{\partial}{\partial x_{2}}+\bar{\sigma} \sin \beta \frac{\partial}{\partial x_{3}}+\bar{\sigma} \cos \beta \frac{\partial}{\partial x_{4}}+\bar{\sigma} \sqrt{\frac{p \sigma}{q} \frac{\partial}{\partial x_{5}}} \\
J Z_{2}=\sigma Z_{2}, J Z_{3}=\bar{\sigma} Z_{3},
\end{gathered}
$$

we remark that $\left\langle J Z_{1}, Z_{k}\right\rangle=0$, for any $k \in\{1,2,3\}, J Z_{2}, J Z_{3} \subseteq \operatorname{span}\left\{Z_{2}, Z_{3}\right\}$ and

$$
\begin{gathered}
\left\|Z_{1}\right\|^{2}=\frac{p \sigma}{q}+2, \quad\left\|Z_{2}\right\|^{2}=\left\|Z_{3}\right\|^{2}=f^{2}, \\
\left\|J Z_{1}\right\|^{2}=p^{2}+2 q-p \bar{\sigma}, \quad\left\|J Z_{2}\right\|^{2}=f^{2}(p \sigma+q), \quad\left\|J Z_{3}\right\|^{2}=f^{2}(p \bar{\sigma}+q) .
\end{gathered}
$$

If $D_{1}:=\operatorname{span}\left\{Z_{1}\right\}$ and $D_{2}:=\operatorname{span}\left\{Z_{2}, Z_{3}\right\}$, then $D_{1}$ is an anti-invariant and $D_{2}$ is an invariant distribution.

Let $M_{\perp}$ and $M_{\top}$ be the integral manifolds of the distributions $D_{1}$ and $D_{2}$, respectively. Thus, $M_{\perp} \times_{f} M_{\top}$ is a warped product semi-invariant submanifold in the metallic Riemannian manifold $\left(R^{5},\langle\cdot, \cdot\rangle, J\right)$, with the metric

$$
g:=g_{M_{\perp}}+f^{2} g_{M_{\top}},
$$

where $g_{M_{\perp}}:=\left(\frac{p \sigma}{q}+2\right) d f^{2}$ and $g_{M_{\top}}:=d \alpha^{2}+d \beta^{2}$.

Proposition 36 ([10]). If $M_{\top} \times_{f} M_{\theta}$ is a proper warped product semi-slant submanifold in a locally metallic Riemannian manifold $(\bar{M}, \bar{g}, J)$, then it is a trivial warped product Riemannian manifold.

We provide an example of a non-trivial warped product semi-slant submanifold $M_{\theta} \times_{f} M_{\top}$ in a metallic Riemannian manifold.

Example 5. We consider the Euclidean space $R^{7}$ endowed with the Euclidean metric $\langle\cdot, \cdot\rangle$ and the immersion $i: M \rightarrow R^{7}$, given by

$$
i(f, \alpha, \beta):=(f \cos \alpha, f \sin \alpha, f \cos \beta, f \sin \beta, f, \alpha, \beta),
$$

where $M:=\left\{(f, \alpha, \beta) \mid f>0, \alpha, \beta \in\left[0, \frac{\pi}{2}\right]\right\}$. 
The local orthogonal frame on TM is

$$
\begin{gathered}
Z_{1}=\cos \alpha \frac{\partial}{\partial x_{1}}+\sin \alpha \frac{\partial}{\partial x_{2}}+\cos \beta \frac{\partial}{\partial x_{3}}+\sin \beta \frac{\partial}{\partial x_{4}}+\frac{\partial}{\partial x_{5}}, \\
Z_{2}=-f \sin \alpha \frac{\partial}{\partial x_{1}}+f \cos \alpha \frac{\partial}{\partial x_{2}}+\frac{\partial}{\partial x_{6}}, Z_{3}=-f \sin \beta \frac{\partial}{\partial x_{3}}+f \cos \beta \frac{\partial}{\partial x_{4}}+\frac{\partial}{\partial x_{7}} .
\end{gathered}
$$

The structure $J: R^{7} \rightarrow R^{7}$ given by

$$
J\left(\frac{\partial}{\partial x_{k}}\right):= \begin{cases}\sigma \frac{\partial}{\partial x_{k}}, & k \in\{1,2,6\} \\ \bar{\sigma} \frac{\partial}{\partial x_{k}}, & k \in\{3,4,5,7\}\end{cases}
$$

is a metallic structure, where $\sigma:=\sigma_{p, q}$ is a metallic number and $\bar{\sigma}:=p-\sigma$. Since

$$
\begin{gathered}
J Z_{1}=\sigma \cos \alpha \frac{\partial}{\partial x_{1}}+\sigma \sin \alpha \frac{\partial}{\partial x_{2}}+\bar{\sigma} \cos \beta \frac{\partial}{\partial x_{3}}+\bar{\sigma} \sin \beta \frac{\partial}{\partial x_{4}}+\bar{\sigma} \frac{\partial}{\partial x_{5}} \\
J Z_{2}=\sigma Z_{2}, J Z_{3}=\bar{\sigma} Z_{3},
\end{gathered}
$$

we remark that $\left\langle J Z_{k}, Z_{l}\right\rangle=0$, for any $k \neq l$, where $k, l \in\{1,2,3\},\left\langle J Z_{1}, Z_{1}\right\rangle=\sigma+2 \bar{\sigma}$ and

$$
\begin{gathered}
\left\|Z_{1}\right\|^{2}=3, \quad\left\|Z_{2}\right\|^{2}=\left\|Z_{3}\right\|^{2}=f^{2}+1 \\
\left\|J Z_{1}\right\|^{2}=\sigma^{2}+2 \bar{\sigma}^{2}, \quad\left\|J Z_{2}\right\|^{2}=\sigma^{2}\left(f^{2}+1\right), \quad\left\|J Z_{3}\right\|^{2}=\bar{\sigma}^{2}\left(f^{2}+1\right) .
\end{gathered}
$$

If $D_{1}:=\operatorname{span}\left\{Z_{1}\right\}$ and $D_{2}:=\operatorname{span}\left\{Z_{2}, Z_{3}\right\}$, then $D_{1}$ is a slant distribution with the slant angle $\theta$ with $\cos \theta=\frac{\sigma+2 \bar{\sigma}}{\sqrt{3\left(\sigma^{2}+2 \bar{\sigma}^{2}\right)}}$, and $D_{2}$ is an invariant distribution.

Let $M_{\theta}$ and $M_{\top}$ be the integral manifolds of the distributions $D_{1}$ and $D_{2}$, respectively. Thus, $M_{\theta} \times \sqrt{f^{2}+1} M_{\top}$ is a warped product semi-slant submanifold in the metallic Riemannian manifold $\left(R^{7},\langle\cdot, \cdot\rangle, J\right)$, with the metric

$$
\begin{aligned}
& \quad g:=g_{M_{\theta}}+\left(f^{2}+1\right) g_{M_{\top}}, \\
& \text { where } g_{M_{\theta}}:=3 d f^{2} \text { and } g_{M_{\top}}:=d \alpha^{2}+d \beta^{2}
\end{aligned}
$$

Proposition 37 ([10]). If $M_{\perp} \times_{f} M_{\theta}$ (or $M_{\theta} \times_{f} M_{\perp}$ ) is a proper warped product hemi-slant submanifold in a locally metallic Riemannian manifold $(\bar{M}, \bar{g}, J)$, then it is a trivial warped product Riemannian manifold if and only if

$$
A_{N Z} X=A_{N X} Z
$$

for any $X \in \Gamma\left(T M_{\perp}\right)$ and $Z \in \Gamma\left(T M_{\theta}\right)$ (or $X \in \Gamma\left(T M_{\theta}\right)$ and $Z \in \Gamma\left(T M_{\perp}\right)$, respectively).

We provide an example of a non-trivial warped product hemi-slant submanifold in a metallic Riemannian manifold.

Example 6. We consider the Euclidean space $R^{5}$ endowed with the Euclidean metric $\langle\cdot, \cdot\rangle$ and let $i: M \rightarrow R^{5}$ be the immersion, given by

$$
i(f, \alpha):=(\sqrt{q} f \sin \alpha, \sqrt{q} f \cos \alpha, \sigma f \sin \alpha, \sigma f \cos \alpha,-\sqrt{q} f),
$$

where $M:=\left\{(f, \alpha) \mid f>0, \alpha \in\left(0, \frac{\pi}{2}\right)\right\}$ and $\sigma:=\sigma_{p, q}$ is a metallic number.

The local orthogonal frame on TM is

$$
\begin{gathered}
Z_{1}=\sqrt{q} \sin \alpha \frac{\partial}{\partial x_{1}}+\sqrt{q} \cos \alpha \frac{\partial}{\partial x_{2}}+\sigma \sin \alpha \frac{\partial}{\partial x_{3}}+\sigma \cos \alpha \frac{\partial}{\partial x_{4}}-\sqrt{q} \frac{\partial}{\partial x_{5}} \\
Z_{2}=\sqrt{q} f \cos \alpha \frac{\partial}{\partial x_{1}}-\sqrt{q} f \sin \alpha \frac{\partial}{\partial x_{2}}+\sigma f \cos \alpha \frac{\partial}{\partial x_{3}}-\sigma f \sin \alpha \frac{\partial}{\partial x_{4}}
\end{gathered}
$$


The structure $J: R^{5} \rightarrow R^{5}$ given by

$$
J\left(\frac{\partial}{\partial x_{k}}\right):= \begin{cases}\sigma \frac{\partial}{\partial x_{k}}, & k \in\{1,2,5\} \\ \bar{\sigma} \frac{\partial}{\partial x_{k}}, & k \in\{3,4\}\end{cases}
$$

is a metallic structure, where $\bar{\sigma}:=1-\sigma$. Since

$$
\begin{gathered}
J Z_{1}=\sqrt{q} \sigma\left(\sin \alpha \frac{\partial}{\partial x_{1}}+\cos \alpha \frac{\partial}{\partial x_{2}}\right)-q\left(\sin \alpha \frac{\partial}{\partial x_{3}}+\cos \alpha \frac{\partial}{\partial x_{4}}\right)-\sqrt{q} \sigma \frac{\partial}{\partial x_{5}}, \\
J Z_{2}=\sqrt{q} \sigma f\left(\cos \alpha \frac{\partial}{\partial x_{1}}-\sin \alpha \frac{\partial}{\partial x_{2}}\right)-q f\left(\cos \alpha \frac{\partial}{\partial x_{3}}-\sin \alpha \frac{\partial}{\partial x_{4}}\right),
\end{gathered}
$$

we remark that $\left\langle J Z_{2}, Z_{k}\right\rangle=0$, for any $k \in\{1,2\},\left\langle J Z_{1}, Z_{1}\right\rangle=q \sigma$ and

$$
\begin{gathered}
\left\|Z_{1}\right\|^{2}=\sigma^{2}+2 q, \quad\left\|Z_{2}\right\|^{2}=f^{2}\left(\sigma^{2}+q\right), \\
\left\|J Z_{1}\right\|^{2}=q\left(2 \sigma^{2}+q\right), \quad\left\|J Z_{2}\right\|^{2}=q f^{2}\left(\sigma^{2}+q\right) .
\end{gathered}
$$

If $D_{1}:=\operatorname{span}\left\{Z_{1}\right\}$ and $D_{2}:=\operatorname{span}\left\{Z_{2}\right\}$, then $D_{1}$ is a slant distribution with the slant angle $\theta$ with $\cos \theta=\frac{\sqrt{q} \sigma}{\sqrt{\left(2 \sigma^{2}+q\right)\left(\sigma^{2}+2 q\right)}}$, and $D_{2}$ is an anti-invariant distribution.

Let $M_{\theta}$ and $M_{\perp}$ be the integral manifolds of the distributions $D_{1}$ and $D_{2}$, respectively. Thus, $M_{\theta} \times_{f \sqrt{\sigma^{2}+q}} M_{\perp}$ is a warped product hemi-slant submanifold in the metallic Riemannian manifold $\left(R^{5},\langle\cdot, \cdot\rangle, J\right)$, with the metric

$$
g=g_{M_{\theta}}+f^{2}\left(\sigma^{2}+q\right) g_{M_{\perp}}
$$

where $g_{M_{\theta}}:=\left(\sigma^{2}+2 q\right) d f^{2}$ and $g_{M_{\perp}}:=d \alpha^{2}$.

Author Contributions: Both authors have contributed to the conceptualization and investigation. Both authors have read and agreed to the published version of the manuscript.

Funding: The APC was funded by Stefan cel Mare University of Suceava, Romania.

Institutional Review Board Statement: Not applicable.

Informed Consent Statement: Not applicable.

Data Availability Statement: Not applicable.

Conflicts of Interest: The authors declare no conflict of interest. The funder had no role in the design of the study; in the collection, analyses, or interpretation of data; in the writing of the manuscript, or in the decision to publish the results.

\section{References}

1. Hretcanu, C.E.; Crasmareanu, M. On some invariant submanifolds in a Riemannian manifold with Golden structure. Analele Stiintifice ale Universitatii Al I Cuza din Iasi 2007, 53, 199-211.

2. Hretcanu, C.E.; Crasmareanu, M.C. Applications of the Golden ratio on Riemannian manifolds. Turk. J. Math. $2009,33,179-191$.

3. Crasmareanu, M.; Hretcanu, C.E.; Munteanu, M.I. Golden- and product-shaped hypersurfaces in real space forms. Int. J. Geom. Methods Mod. Phys. 2013, 10, 1320006. [CrossRef]

4. Hretcanu, C.E.; Crasmareanu, M. Metallic structures on Riemannian manifolds. Revista de la Unión Matemática Argentina 2013, 54, 15-27.

5. Blaga, A.M.; Hretcanu, C.E. Invariant, anti-invariant and slant submanifolds of a metallic Riemannian manifold. Novi Sad J. Math. 2018, 48, 55-80. [CrossRef]

6. Hretcanu, C.E.; Blaga, A.M. Slant and semi-slant submanifolds in metallic Riemannian manifolds. J. Funct. Spaces 2018, 2018, 2864263. [CrossRef]

7. Hretcanu, C.E.; Blaga, A.M. Hemi-slant submanifolds in metallic Riemannian manifolds. Carpathian J. Math. 2019, 35, 59-68. [CrossRef]

8. Blaga, A.M.; Hretcanu, C.E. Golden warped product Riemannian manifolds. Lib. Math. 2017, 37, 39-49. 
9. Blaga, A.M.; Hretcanu, C.E. Remarks on metallic warped product manifolds. Facta Univ. Ser. Math. Inform. 2018, 33, $269-277$.

10. Hretcanu, C.E.; Blaga, A.M. Warped product submanifolds in metallic Riemannian manifods. Tamkang J. Math. 2020, 51, 161-186. [CrossRef]

11. Hretcanu, C.E.; Blaga, A.M. Submanifolds in metallic Riemannian manifolds. Differ. Geom. Dyn. Syst. 2018, $20,83-97$.

12. De Spinadel, V.W. The metallic means family and forbidden symmetries. Int. Math. J. 2002, 2, $279-288$.

13. De Spinadel, V.W. The metallic means family and renormalization group techniques. Trudy Instituta Matematiki i Mekhaniki UrO RAN 2000, 6, 173-189.

14. De Spinadel, V.W. A New Family of Irrational Numbers with Curious Properties. Humanist. Math. Netw. J. 1999, 19, 133-37. [CrossRef]

15. De Spinadel, V.W. The metallic means family and multifractal spectra. Nonlinear Anal. 1999, 36, 721-745. [CrossRef]

16. Stakhov, A.P. The generalized Golden proportions, a new theory of real numbers, and ternary mirror-symmetrical arithmetic. Chaos Solitons Fractals 2007, 33, 315-334. [CrossRef]

17. Hretcanu, C.E.; Blaga, A.M.; Hretcanu, C.I. On some generalisations of the Golden proportion and of the Golden rectangle. Food Eng. 2009, 1, 63-69.

18. Livio, M. The Golden Ratio: The Story of Phi, the World's Most Astonishing Number; Broadway Books: New York, NY, USA, 2002.

19. Falcon, S.; Plaza, A. On 3-dimensional $k$-Fibonacci spirals. Chaos Solitons Fractals 2008, 38, 993-1003. [CrossRef]

20. Naschie, M.S.E. COBE satellite measurement, hyperspheres, superstrings and the dimension of space time. Chaos Solitons Fractals 1998, 9, 1445-1471. [CrossRef]

21. Naschie, M.S.E. The Golden mean in quantum geometry, knot theory and related topics. Chaos Solitons Fractals 1999, 10, $1303-1307$. [CrossRef]

22. Goldberg, S.I.; Yano, K. Polynomial structures on manifolds. Kodai Math. Semin. Rep. 1970, 22, 199-218. [CrossRef]

23. Goldberg, S.I.; Petridis, N.C. Differentiable solutions of algebraic equations on manifolds. Kodai Math. Semin. Rep. 1973, 25, 111-128. [CrossRef]

24. Crasmareanu, M.; Hretcanu, C.E. Golden differential geometry. Chaos Solitons Fractals 2008, 38, 1229-1238. [CrossRef]

25. Etayo, F.; Santamaria, R. Classification of Almost Golden Riemannian Manifolds with Null Trace. Mediterr. J. Math. 2020, 17, 90. [CrossRef]

26. Yano, K.; Kon, M. Structures on Manifolds; Series in Pure Mathematics; World Scientific: Singapore, 1985; Volume 3.

27. Mihai, I.; Roşca, R.; Verstraelen, L. Some Aspects of the Differential Geometry of Vector Fields; Katholicke Universiteit Leuven, PADGE: Leuven, Belgium, 1996; Volume 2.

28. Li, H.; Liu, X. Semi-slant submanifolds of a locally product manifold. Georgian Math. J. 2005, 12, 273-282. [CrossRef]

29. Chen, B.-Y. Geometry of Slant Submanifolds; Katholieke Universiteit Leuven: Leuven, Belgium, 1990; 123p.

30. Atçeken, M. Slant submanifolds of a Riemannian product manifold. Acta Math. Sci. Ser. B Engl. Ed. 2010, 30, 215-224. [CrossRef]

31. SSahin, B. Slant submanifolds of an almost product Riemannian manifold. J. Korean Math. Soc. 2006, 43, 717-732. [CrossRef]

32. Cabrerizo, J.L.; Carriazo, A.; Fernandez, L.M.; Fernandez, M. Slant submanifolds in Sasakian manifolds. Glasg. Math. J. 2000, 42, 125-138. [CrossRef]

33. Cabrerizo, J.L.; Carriazo, A.; Fernandez, L.M.; Fernandez, M. Semi-slant submanifolds of a Sasakian manifold. Geom. Dedicata 1999, 78, 183-199. [CrossRef]

34. Carriazo, A. New Developments in Slant Submanifolds Theory; Narasa Publishing House: New Delhi, India, 2002.

35. Atçeken, M.; Dirik, S.; Yildirim, U. Pseudo-slant submanifolds of a locally decomposable Riemannian manifold. J. Adv. Math. 2015, 11, 5587-5599. [CrossRef]

36. Taştan, H.M.; Ozdem, F. The geometry of hemi-slant submanifolds of a locally product Riemannian manifold. Turk. J. Math. 2015, 39, 268-284. [CrossRef]

37. Chen, B.-Y. Differential Geometry of Warped Product Manifolds and Submanifolds; World Scientific: Singapore, 2017.

38. Al-Solamy, F.R.; Khan, M.A. Warped product submanifolds of Riemannian product manifolds. Abstr. Appl. Anal. 2012, 2012, 724898. [CrossRef]

39. Al-Solamy, F.R.; Uddin, S. An inequality for warped product submanifolds of a locally product Riemannian manifold. Hacet. J. Math. Stat. 2019, 48, 351-358. [CrossRef]

40. Uddin, S.; Ali, A.; Al-Asmari, N.M.; Othman, W.A. Warped product pseudo-slant submanifolds in a locally product Riemannian manifold. Differ. Geom. Dyn. Syst. 2016, 18, 147-158.

41. Şahin, B. Warped product semi-slant submanifolds of a locally product Riemannian manifold. Stud. Sci. Math. Hung. 2009, 46, 169-184.

42. Atçeken, M. Warped product semi-invariant submanifolds in locally decomposable Riemannian manifold. Hacet. J. Math. Stat. 2011, 40, 401-407.

43. Atçeken, M. Geometry of warped product semi-invariant submanifolds of a locally Riemannian product manifold. Serdica Math. J. 2009, 35, 273-286.

44. Atçeken, M. Warped product semi-slant submanifolds in locally Riemannian product manifolds. Bull. Aust. Math. Soc. 2008, 77, 177-186. [CrossRef]

45. Bishop, R.L.; O'Neill, B. Manifolds of negative curvature. Trans. Am. Math. Soc. 1969, 145, 1-49. [CrossRef] 